CRYSTALLOGRAPHIC COMMUNICATIONS

ISSN 2056-9890

Received 12 January 2022

Accepted 1 February 2022

Edited by D. Chopra, Indian Institute of Science Education and Research Bhopal, India

Keywords: crystal structure; fluoroquinolone; ciprofloxacin; dihydroxybenzoic acid; salt cocrystal; antibiotic.

CCDC references: 2098049; 2098403

Supporting information: this article has supporting information at journals.iucr.org/e

\section{Ciprofloxacin salt and salt co-crystal with dihydroxybenzoic acids}

\author{
Yuda Prasetya Nugraha, ${ }^{a *}$ Haruki Sugiyama ${ }^{b, c}$ and Hidehiro Uekusa ${ }^{d}$
}

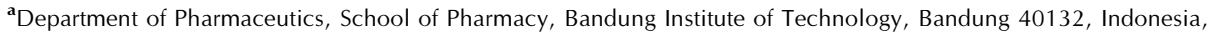
${ }^{\mathbf{b}}$ Research and Education Center for Natural Sciences, Keio University, Hiyoshi, 4-1-1, Kohoku, Yokohama 223-8521, Japan, 'Department of Life and Coordination-Complex Molecular Science, Institute for Molecular Science, Myodaiji, Okazaki 444-8787, Japan, and d Department of Chemistry, Tokyo Institute of Technology, 2-12-1, Ookayama, Meguro, Tokyo 152-8551, Japan. *Correspondence e-mail: yudapn@itb.ac.id

The crystal structure of two multi-component crystals of ciprofloxacin [systematic name: 1-cyclopropyl-6-fluoro-4-oxo-7-(piperazin-1-yl)quinoline-3carboxylic acid], a fluoroquinolone antibiotic, namely, ciprofloxacin 2,6dihydroxybenzoate salt, $\mathrm{C}_{17} \mathrm{H}_{19} \mathrm{FN}_{3} \mathrm{O}_{3}{ }^{+} \cdot \mathrm{C}_{7} \mathrm{H}_{5} \mathrm{O}_{4}{ }^{-}$, (I), and ciprofloxacin hydrochloride-3,5-dihydroxybenzoic-water (1/1/1), $\mathrm{C}_{17} \mathrm{H}_{19} \mathrm{FN}_{3} \mathrm{O}_{3}{ }^{+} \cdot \mathrm{Cl}^{-} \cdot \mathrm{C}_{7} \mathrm{H}_{6} \mathrm{O}_{4} \cdot \mathrm{H}_{2} \mathrm{O}$, (II), were determined. In (I) and (II), the ciprofloxacin cations are connected via head-to-tail $\mathrm{N}-\mathrm{H} \cdots \mathrm{O}$ hydrogen bonding. Both structures show an alternating layered arrangement between ciprofloxacin and dihydroxybenzoic acid.

\section{Chemical context}

The design and exploration of multi-component crystals of active pharmaceutical ingredients (APIs) have gained increasing interest over recent decades. The formation of multi-component crystals, i.e. salts and co-crystals through a crystal-engineering approach has been continuously demonstrated as a versatile tool to improve the physicochemical properties of APIs (Kavanagh et al., 2019; Putra \& Uekusa, 2020; Thakur \& Thakuria, 2020). Recently, the co-crystallization of salt APIs or salt co-crystal formation has been increasingly studied. Salt co-crystallization has been utilized to suppress hydrate formation of salt APIs (Nugraha \& Uekusa, 2018; Fujito et al., 2021). As a part of our study of salt cocrystals of APIs, we investigated multi-component crystals of ciprofloxacin. Ciprofloxacin is a Biopharmaceutics Classification System (BCS) class IV fluoroquinolone antibiotic that is widely used therapeutically as the free base and the hydrochloride salt (Olivera et al., 2011).

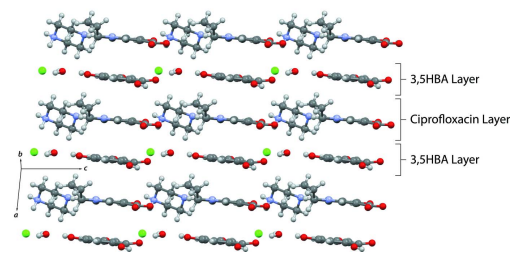

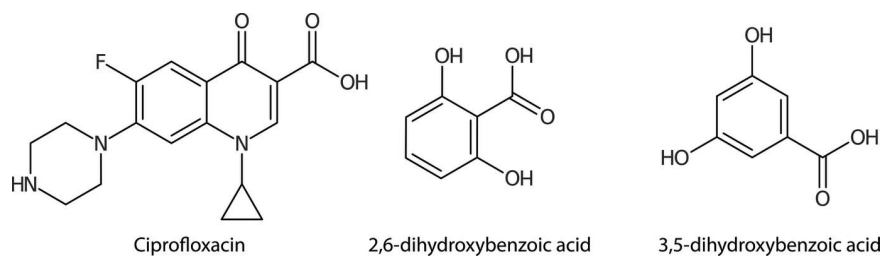

\section{Structural commentary}

Compound (I) was obtained as an anion-exchange product between ciprofloxacin hydrochloride and 2,6-dihydrobenzoic acid in solution. 2,6-Dihydroxybenzoic acid (2,6HBA) is a 


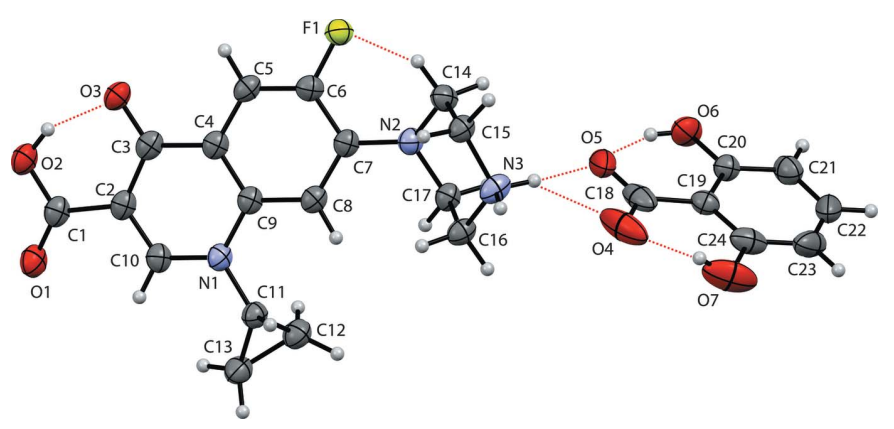

Figure 1

Displacement ellipsoid (50\% probability level) drawing with the atomic labelling scheme for compound (I) showing the hydrogen bonds within the selected asymmetric unit.

relatively strong carboxylic acid with a $K_{\mathrm{a}}$ of 1.30 (Gdaniec et al., 1994; Habibi-yangjeh et al., 2005). Compound (I) crystallizes in the monoclinic space group $P 2_{1} / c$. The asymmetric unit consists of one ciprofloxacin cation and one 2,6 HBA anion (Fig. 1). The $\mathrm{C}-\mathrm{O}$ distances of the ciprofloxacin carboxylic group i.e., 1.218 (3) and 1.325 (3) $\AA$ indicate that it exists as the neutral carboxylic form. However, in 2,6HBA, the $\mathrm{C}-\mathrm{O}$ distances are very similar i.e., 1.263 (4) and 1.267 (3) $\AA$ due to resonance stabilization in the carboxylate anion (Childs et al., 2007; Aakeröy et al., 2006). As a result, the piperazinyl group of ciprofloxacin is protonated. Therefore, compound (I) is a salt. The formation of a salt is well-predicted by the $\mathrm{p} K_{\mathrm{a}}$ rule (Cruz-Cabeza, 2012). The $\mathrm{p} K_{\mathrm{a}}$ of ciprofloxacin are 6.18 and 8.73 for the carboxylic acid and the piperazinyl ring, respectively (Sun et al., 2002). Therefore, salt formation is expected because the $\Delta \mathrm{p} K_{\mathrm{a}}$ between the piperazinyl ring of ciprofloxacin and the carboxylic acid of 2,6HBA is greater than 4 . Similar behaviour is observed in the salicylate salt of ciprofloxacin (Surov et al., 2019; Nugrahani et al., 2020).

Compound (II) crystallizes in the non-centrosymmetric $P 1$ space group despite the lack of a chiral centre. The asymmetric unit comprises one ciprofloxacin cation, one chloride anion and one 3,5HBA molecule, as shown in Fig. 2. In addition, one water molecule is incorporated into the crystal lattice. An anion-exchange reaction during crystallization did not occur in this system. Compared to 2,6HBA, the coformer is a weaker acid with a $\mathrm{p} K_{\mathrm{a}}$ of 4.04 (Habibi-yangjeh et al., 2005). Contrary to the previous structures, the coformer exists as a neutral molecule in the crystal. The carboxylic $\mathrm{C} 18-\mathrm{O} 4$ and $\mathrm{C} 18-\mathrm{O} 5$ distances of 2,6HBA are 1.320 (4) and 1.216 (4) $\AA$, respec-

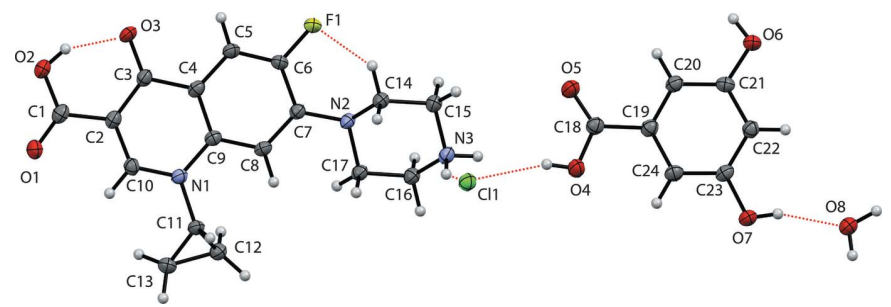

Figure 2

Displacement ellipsoid (50\% probability level) drawing with the atomic labelling scheme for compound (II) showing the hydrogen bonds within the selected asymmetric unit.

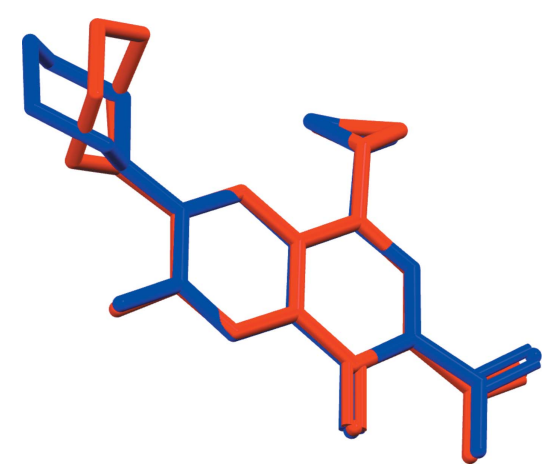

Figure 3

Molecular overlay of ciprofloxacin cation in compounds (I) (red) and (II) (blue). Hydrogen atoms are omitted for clarity.

tively, confirming its neutral state. Additionally, the carboxylic $\mathrm{C} 1-\mathrm{O} 1$ and $\mathrm{C} 1-\mathrm{O} 2$ distances of ciprofloxacin, i.e. 1.227 (4) and 1.314 (4) $\AA$, respectively, also confirm the neutral state of this moiety. On the other hand, the piperazinyl group is protonated. Hence, compound (II) is a salt co-crystal monohydrate of ciprofloxacin.

Compounds (I) and (II) exhibit similar conformations, as shown in Fig. 3. The molecular conformation of the ciprofloxacin molecule is governed by intramolecular $\mathrm{O} 2-$ $\mathrm{H} 2 \cdots \mathrm{O} 3$ and $\mathrm{C} 14-\mathrm{H} 14 A \cdots \mathrm{F} 1$ hydrogen bonding (Tables 1 and 2). In both structures, the piperazinium ring exhibits a chair conformation. The main difference is the relative orientation between the piperazinium moiety and the quinolone ring. The $\mathrm{C} 7-\mathrm{N} 2-\mathrm{C} 14-\mathrm{C} 15$ torsion angles are 97.0 (2) and $-167.8(2)^{\circ}$, respectively, for compounds (I) and (II).

\section{Supramolecular features}

In compound (I), the carboxylate anion of 2,6HBA acts as a hydrogen-bond donor for intramolecular hydrogen bonds involving two hydroxyl groups, namely $\mathrm{O} 6-\mathrm{H} 6 \cdots \mathrm{O} 5$ and $\mathrm{O} 7-\mathrm{H} 7 \cdots \mathrm{O} 4$. The protonated nitrogen atom $\mathrm{N} 3$ of the piperazinyl ring is involved in the formation of trifurcated hydrogen bonds with $\mathrm{O} 4, \mathrm{O} 5$, and $\mathrm{O} 6$ of the coformer. These charge-assisted hydrogen bonds, i.e. $\mathrm{N} 3-\mathrm{H} 3 B \cdots \mathrm{O} 4, \mathrm{~N} 3-$

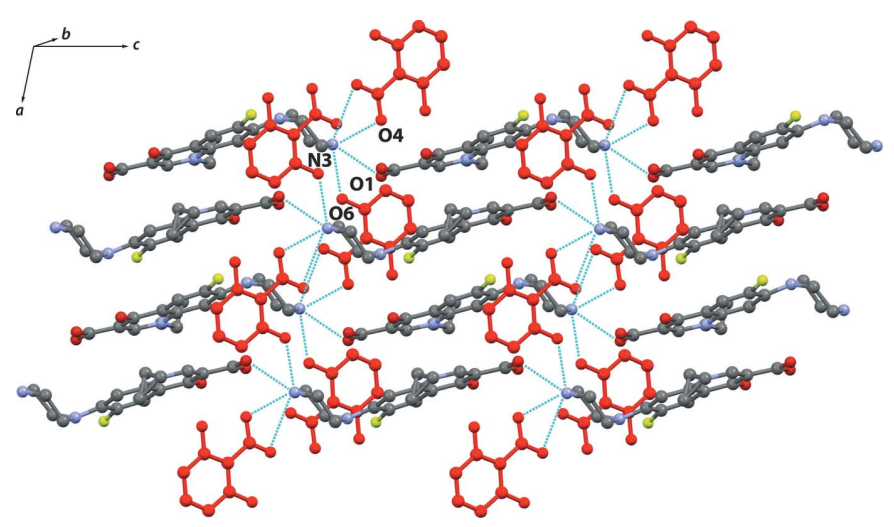

Figure 4

Intermolecular hydrogen-bonding motifs in (I) showing infinite chains along the $a$-axis direction formed by ciprofloxacin and 2,6HBA (red). Hydrogen atoms are omitted for clarity. 
$\mathrm{H} 3 B \cdots \mathrm{O} 5$, and $\mathrm{N} 3-\mathrm{H} 3 A \cdots \mathrm{O} 6$, form an infinite chain structure along the $a$-axis direction (Table 1, Fig. 4). The chains are connected to the adjacent ciprofloxacin molecule through head-to-tail $\mathrm{N} 3-\mathrm{H} 3 A \cdots \mathrm{O} 1$ hydrogen bonding. The crystal packing of (I) is shown in Fig. 5. Along the $a$-axis, centrosymmetric pairs of ciprofloxacin molecules are stacked by $\pi-\pi$ interactions. The distance between the centroids of symmetryrelated C4-C9 rings is 3.4986 (11) $\AA$. This arrangement leads to the formation of a columnar packing arrangement. Interestingly, a similar packing feature was observed in the 1.75

(a)

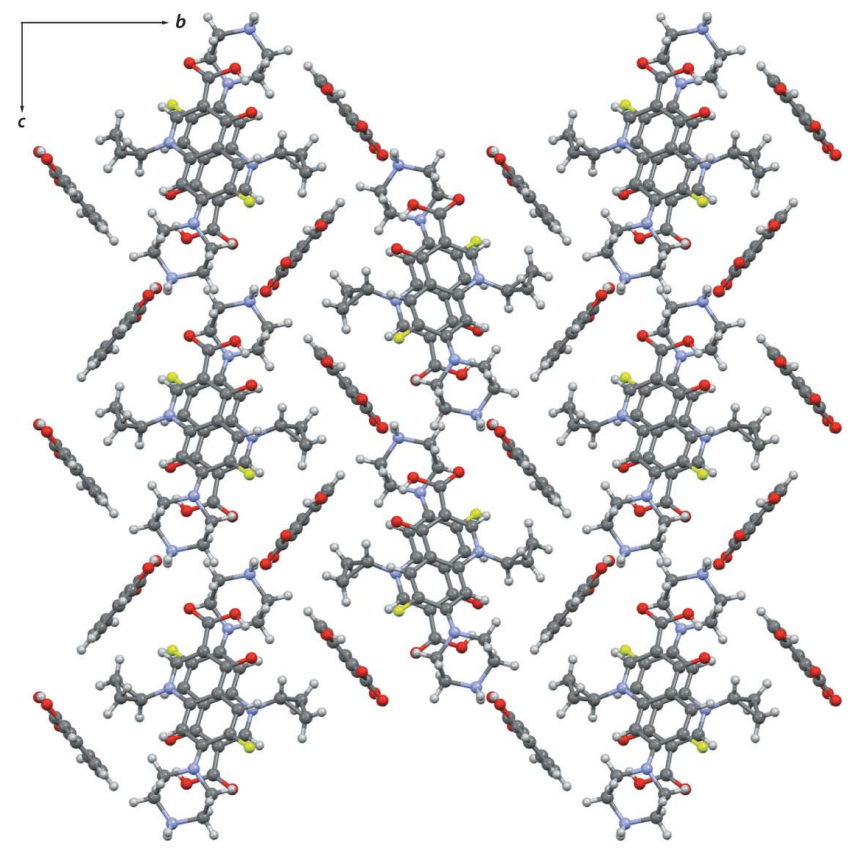

(b)

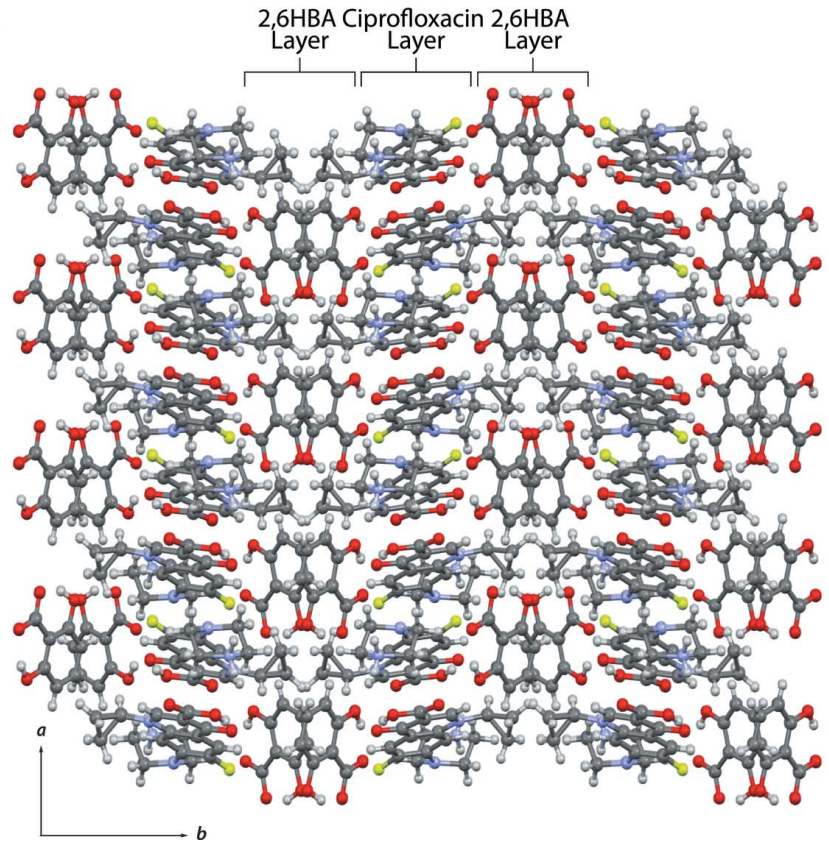

Figure 5

Packing motifs of (I) viewed along (a) the $a$ axis and (b) the $c$ axis highlighting the alternating layers of ciprofloxacin and the coformer.
Table 1

Hydrogen-bond geometry $\left(\AA,^{\circ}\right)$ for (I).

\begin{tabular}{|c|c|c|c|c|}
\hline$D-\mathrm{H} \cdots A$ & $D-\mathrm{H}$ & $\mathrm{H} \cdots A$ & $D \cdots A$ & $D-\mathrm{H} \cdots A$ \\
\hline $\mathrm{O} 2-\mathrm{H} 2 \cdots \mathrm{O} 3$ & 0.84 & 1.73 & $2.512(2)$ & 155 \\
\hline $\mathrm{N} 3-\mathrm{H} 3 A \cdots \mathrm{O} 1^{\mathrm{i}}$ & 0.91 & 2.38 & $2.977(2)$ & 123 \\
\hline $\mathrm{N} 3-\mathrm{H} 3 A \cdots \mathrm{O} 6$ & 0.91 & 2.09 & $2.890(2)$ & 146 \\
\hline $\mathrm{N} 3-\mathrm{H} 3 B \cdots \mathrm{O} 4^{\mathrm{ii}}$ & 0.91 & 2.18 & $2.897(3)$ & 136 \\
\hline $\mathrm{N} 3-\mathrm{H} 3 B \cdots \mathrm{O} 5^{\mathrm{ii}}$ & 0.91 & 2.24 & $3.090(3)$ & 155 \\
\hline $\mathrm{C} 11-\mathrm{H} 11 \cdots \mathrm{O} 3^{\mathrm{iii}}$ & 1.00 & 2.46 & $3.239(3)$ & 134 \\
\hline $\mathrm{C} 12-\mathrm{H} 12 A \cdots \mathrm{O} 4^{\mathrm{iv}}$ & 0.99 & 2.54 & $3.374(3)$ & 141 \\
\hline $\mathrm{C} 13-\mathrm{H} 13 A \cdots \mathrm{O} 7^{\mathrm{v}}$ & 0.99 & 2.51 & $3.193(3)$ & 126 \\
\hline $\mathrm{C} 14-\mathrm{H} 14 A \cdots \mathrm{F} 1$ & 0.99 & 2.13 & $2.831(2)$ & 126 \\
\hline $\mathrm{C} 15-\mathrm{H} 15 B \cdots \mathrm{O} 1^{\mathrm{iii}}$ & 0.99 & 2.33 & $3.282(3)$ & 161 \\
\hline $\mathrm{C} 17-\mathrm{H} 17 A \cdots \mathrm{O} 5^{\mathrm{ii}}$ & 0.99 & 2.60 & $3.408(3)$ & 139 \\
\hline 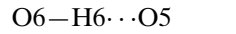 & 0.84 & 1.77 & $2.520(3)$ & 148 \\
\hline $\mathrm{O} 7-\mathrm{H} 7 \cdots \mathrm{O} 4$ & 0.84 & 1.85 & $2.508(4)$ & 134 \\
\hline $\mathrm{C} 21-\mathrm{H} 21 \cdots \mathrm{O} 4^{\mathrm{ii}}$ & 0.95 & 2.54 & $3.488(3)$ & 178 \\
\hline
\end{tabular}

Symmetry codes: (i) $x, y, z+1$; (ii) $x-1, y, z$; (iii) $-x+1,-y+1,-z+1$; (iv) $x-1,-y+\frac{3}{2}, z-\frac{1}{2} ;$ (v) $x-1, y, z-1$.

hydrate of ciprofloxacin salicylate (Nugrahani et al., 2020). In addition, compound (I) shows a layered structure with alternating ciprofloxacin and 2,6HBA layers along the $b$ axis.

The supramolecular features of compound (II) are similar to those observed in compound (I). Ciprofloxacin cations are interconnected through head-to-tail $\mathrm{N} 3-\mathrm{H} 3 A \cdots \mathrm{O} 1$ hydrogen bonds (Table 2), forming an infinite chain arrangement. The chloride ion and water molecule are involved in an extensive hydrogen-bond network bridging ciprofloxacin and 3,5HBA (Fig. 6a). Interestingly, compound (II) also shows a layered arrangement of ciprofloxacin and the coformer (Fig. 6b).

(a)

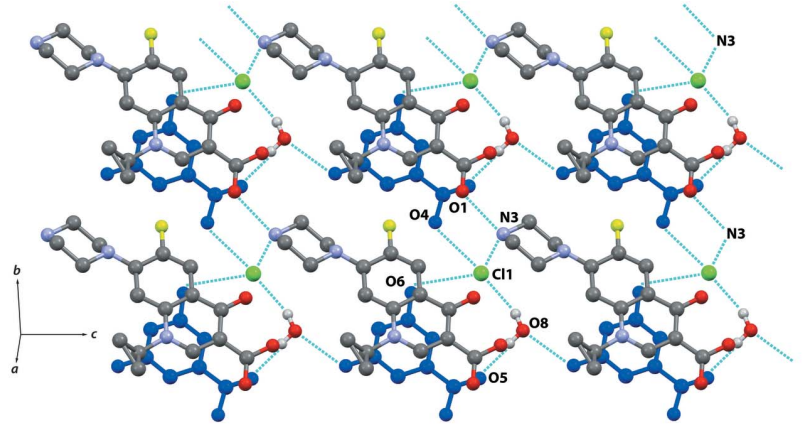

(b)

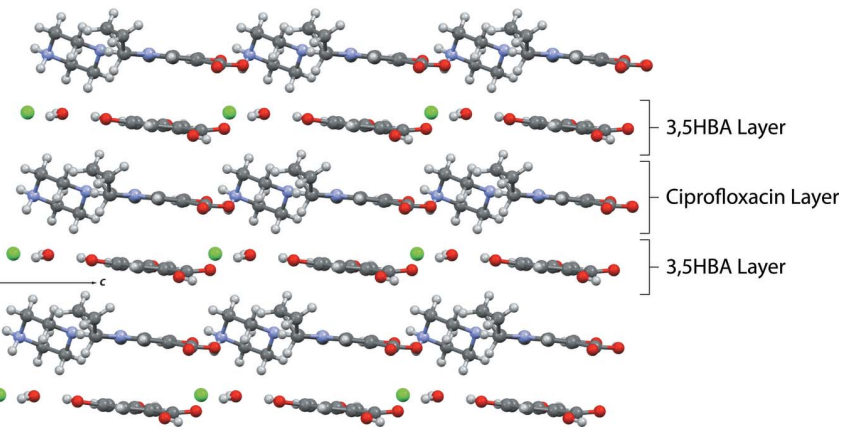

Figure 6

Intermolecular hydrogen-bonding motifs in (II) highlighting the role of the chloride ion and water molecule in bridging ciprofloxacin and 3,5HBA (blue). Hydrogen atoms are omitted for clarity. (b) The crystal packing of (II) showing the alternating layered arrangement. 
Table 2

Hydrogen-bond geometry $\left(\AA{ }^{\circ}{ }^{\circ}\right)$ for (II).

\begin{tabular}{|c|c|c|c|c|}
\hline$D-\mathrm{H} \cdots A$ & $D-\mathrm{H}$ & $\mathrm{H} \cdots A$ & $D \cdots A$ & $D-\mathrm{H} \cdots A$ \\
\hline $\mathrm{O} 2-\mathrm{H} 2 \cdots \mathrm{O} 3$ & 0.84 & 1.78 & $2.551(3)$ & 152 \\
\hline $\mathrm{N} 3-\mathrm{H} 3 A \cdots \mathrm{O} 1^{\mathrm{i}}$ & 0.91 & 1.75 & $2.652(3)$ & 172 \\
\hline $\mathrm{N} 3-\mathrm{H} 3 B \cdots \mathrm{Cl} 1$ & 0.91 & 2.30 & 3.106 (3) & 148 \\
\hline $\mathrm{C} 10-\mathrm{H} 10 \cdots \mathrm{F} 1^{\mathrm{ii}}$ & 0.95 & 2.46 & $3.158(4)$ & 130 \\
\hline $\mathrm{C} 12-\mathrm{H} 12 B \cdots \mathrm{O} 7^{\mathrm{iii}}$ & 0.99 & 2.47 & $3.435(4)$ & 166 \\
\hline $\mathrm{C} 14-\mathrm{H} 14 B \cdots \mathrm{F} 1$ & 0.99 & 2.27 & $2.927(3)$ & 123 \\
\hline $\mathrm{C} 16-\mathrm{H} 16 B \cdots \mathrm{Cl} 1^{\mathrm{iv}}$ & 0.99 & 2.78 & $3.609(3)$ & 142 \\
\hline $\mathrm{O} 4-\mathrm{H} 4 \cdots \mathrm{Cl} 1$ & 0.84 & 2.28 & $3.082(2)$ & 160 \\
\hline $\mathrm{O} 6-\mathrm{H}_{6} \cdots \mathrm{Cl}^{\mathrm{v}}$ & 0.84 & 2.40 & $3.232(2)$ & 170 \\
\hline $\mathrm{O} 7-\mathrm{H} 7 \cdots \mathrm{O} 8$ & 0.84 & 1.96 & $2.769(3)$ & 161 \\
\hline $\mathrm{O} 8-\mathrm{H} 8 A \cdots \mathrm{Cl} 1^{\mathrm{i}}$ & $0.88(6)$ & $2.51(6)$ & $3.362(3)$ & $164(4)$ \\
\hline $\mathrm{O} 8-\mathrm{H} 8 B \cdots \mathrm{O} 5^{\mathrm{vi}}$ & $0.82(6)$ & $2.05(6)$ & $2.865(4)$ & $170(5)$ \\
\hline
\end{tabular}

Symmetry codes: (i) $x, y+1, z-1$; (ii) $x, y-1, z$; (iii) $x-1, y-1, z+1$; (iv) $x-1, y, z$; (v) $x, y+1, z ;(\mathrm{vi}) x, y, z-1$.

\section{Database survey}

Several crystal structures of ciprofloxacin salts with benzoic acid derivatives have been reported, including salts with salicylic acid (Surov et al., 2019; Nagalapalli \& Yaga Bheem, 2014; CSD refcode family DOFWUT), 4-hydroxybenzoic acid (Surov et al., 2020; CSD refcode PUNMUJ), 4-aminobenzoic acid (Surov et al., 2020; CSD refcode PUNMIX) and gallic acid (Surov et al., 2020; CSD refcode PUNMOD). A search for salt co-crystals of ciprofloxacin hydrochloride yielded one reported crystal structure, a co-crystal of ciprofloxacin hydrochloride with 4-hydroxybenzoic acid (Martínez-Alejo et al., 2014; CSD refcode XOHTUL). Compound (II) was also disclosed in a patent without any structural information (Rojas et al., 2016).

\section{Synthesis and crystallization}

Single crystals of (I) and (II) were obtained by preparing a saturated solution of equimolar ciprofloxacin hydrochloride and the respective coformer in methanol/water (1:1) at room temperature. The saturated solution was allowed to slowly evaporate at room temperature. A suitable single crystal was selected and measured for structure determination.

\section{Refinement}

Crystal data, data collection and structure refinement details are summarized in Table 3. All non-hydrogen atoms were refined anisotropically. All hydrogen atoms were refined using a riding model and their displacement parameters $\left(U_{\text {iso }}\right)$ were fixed to $1.2 U_{\text {eq }}$ of the parent carbon or nitrogen atom and $1.5 U_{\text {eq }}$ for hydroxyl groups.

Table 3

Experimental details.

(I)

Crystal data

Chemical formula

$M_{\mathrm{r}}$

Crystal system, space group

Temperature (K)

$a, b, c(\AA)$

$\alpha, \beta, \gamma\left({ }^{\circ}\right)$

$V\left(\AA^{3}\right)$

$Z$

Radiation type

$\mu\left(\mathrm{mm}^{-1}\right)$

Crystal size (mm)

Data collection

Diffractometer

Absorption correction

$T_{\min }, T_{\max }$

No. of measured, independent and observed reflections

$R_{\text {int }}$

$(\sin \theta / \lambda)_{\max }\left(\AA^{-1}\right)$

Refinement

$R\left[F^{2}>2 \sigma\left(F^{2}\right)\right], w R\left(F^{2}\right), S$

No. of reflections

No. of parameters

No. of restraints

$\mathrm{H}$-atom treatment

$\Delta \rho_{\max }, \Delta \rho_{\min }\left(\mathrm{e} \AA^{-3}\right)$

Absolute structure

Absolute structure parameter

$-$

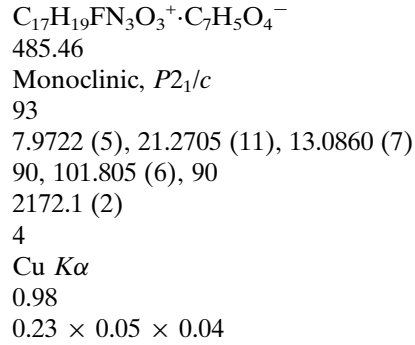

XtaLAB Synergy R, DW system, HyPix $0.919,1.000$

15936, 4378, 3601 (?)

0.038

0.630

$0.053,0.139,1.04$

4378

319

0

$\mathrm{H}$-atom parameters constrained

$0.34,-0.41$

$-$ Multi-scan (CrysAlis PRO; Rigaku OD, 2020)

(II)

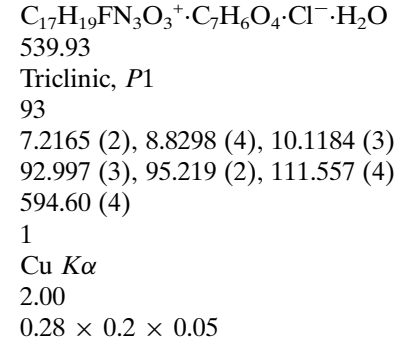

XtaLAB Synergy R, DW system, HyPix Multi-scan (CrysAlis PRO; Rigaku OD, 2020) $0.839,1.000$

$16358,4420,4323[I>2 \sigma(I)]$

0.035

0.625

$0.034,0.094,1.12$
4420
344
3
H atoms treated by a mixture of independent
and constrained refinement
$0.25,-0.47$
Flack $x$ determined using 1889 quotients
$\left[\left(I^{+}\right)-\left(I^{-}\right)\right] /\left[\left(I^{+}\right)+\left(I^{-}\right)\right]$(Parsons et al., 2013)
$0.011(7)$

$0.034,0.094,1.12$

4420

34 $0.25,-0.47$

$\left[\left(I^{+}\right)-\left(I^{-}\right)\right] /\left[\left(I^{+}\right)+\left(I^{-}\right)\right]$(Parsons et al., 2013) 


\section{Acknowledgements}

The authors thank the Materials Analysis Division of the Open Facility Center at the Tokyo Institute of Technology for the research facilities.

\section{References}

Aakeröy, C. B., Hussain, I. \& Desper, J. (2006). Cryst. Growth Des. 6, 474-480.

Childs, S. L., Stahly, G. P. \& Park, A. (2007). Mol. Pharm. 4, 323-338.

Cruz-Cabeza, A. J. (2012). CrystEngComm, 14, 6362-6365.

Dolomanov, O. V., Bourhis, L. J., Gildea, R. J., Howard, J. A. K. \& Puschmann, H. (2009). J. Appl. Cryst. 42, 339-341.

Fujito, T., Oshima, T., Higashi, K., Ueda, K., Ito, M., Masu, H., Noguchi, S. \& Moribe, K. (2021). Cryst. Growth Des. https://doi.org/ 10.1021/acs.cgd.1c01050.

Gdaniec, M., Gilski, M. \& Denisov, G. S. (1994). Acta Cryst. C50, $1622-1626$.

Habibi-yangjeh, A., Danandeh-jenagharad, M. \& Nooshyar, M. (2005). Bull. Korean Chem. Soc. 26, 2007-2016.

Kavanagh, O. N., Croker, D. M., Walker, G. M. \& Zaworotko, M. J. (2019). Drug Discovery Today, 24, 796-804.

Macrae, C. F., Sovago, I., Cottrell, S. J., Galek, P. T. A., McCabe, P., Pidcock, E., Platings, M., Shields, G. P., Stevens, J. S., Towler, M. \& Wood, P. A. (2020). J. Appl. Cryst. 53, 226-235.

Martínez-Alejo, J. M., Domínguez-Chávez, J. G., Rivera-Islas, J., Herrera-Ruiz, D., Höpfl, H., Morales-Rojas, H. \& Senosiain, J. P. (2014). Cryst. Growth Des. 14, 3078-3095.
Nagalapalli, R. \& Yaga Bheem, S. (2014). J. Crystallogr. pp. 1-5.

Nugraha, Y. P. \& Uekusa, H. (2018). CrystEngComm, 20, 2653-2662.

Nugrahani, I., Tjengal, B., Gusdinar, T., Horikawa, A. \& Uekusa, H. (2020). Crystals, 10, 1-19.

Olivera, M. E., Manzo, R. H., Junginger, H. E., Midha, K. K., Shah, V. P., Stavchansky, S., Dressman, J. B. \& Barends, D. M. (2011). J. Pharm. Sci. 100, 22-33.

Parsons, S., Flack, H. D. \& Wagner, T. (2013). Acta Cryst. B69, 249259.

Putra, O. D. \& Uekusa, H. (2020). Advances in Organic Crystal Chemistry, pp. 153-184. Singapore: Springer Singapore.

Rigaku OD (2020). CrysAlis PRO. Rigaku Oxford Diffraction, Yarnton, England.

Rojas, H. M., Chávez, J. G. D., Höpfl, D. H. R., Alejo, J. M. M. \& Peláez, J. P. S. (2016). Solid forms of antibiotics. United States Patent and Trademark Office.

Sheldrick, G. M. (2015a). Acta Cryst. A71, 3-8.

Sheldrick, G. M. (2015b). Acta Cryst. C71, 3-8.

Sun, J., Sakai, S., Tauchi, Y., Deguchi, Y., Chen, J., Zhang, R. \& Morimoto, K. (2002). Eur. J. Pharm. Biopharm. 54, 51-58.

Surov, A. O., Vasilev, N. A., Churakov, A. V., Stroh, J., Emmerling, F. \& Perlovich, G. L. (2019). Cryst. Growth Des. 19, 2979-2990.

Surov, A. O., Vasilev, N. A., Voronin, A. P., Churakov, A. V., Emmerling, F. \& Perlovich, G. L. (2020). CrystEngComm, 22, 42384249.

Thakur, T. S. \& Thakuria, R. (2020). Cryst. Growth Des. 20, 62456265 . 


\section{supporting information}

Acta Cryst. (2022). E78, 259-263 [https://doi.org/10.1107/S2056989022001177]

\section{Ciprofloxacin salt and salt co-crystal with dihydroxybenzoic acids}

\section{Yuda Prasetya Nugraha, Haruki Sugiyama and Hidehiro Uekusa}

\section{Computing details}

For both structures, data collection: CrysAlis PRO (Rigaku OD, 2020); cell refinement: CrysAlis PRO (Rigaku OD, 2020); data reduction: CrysAlis PRO (Rigaku OD, 2020); program(s) used to solve structure: SHELXT2018/2 (Sheldrick, 2015a); program(s) used to refine structure: SHELXL2018/3 (Sheldrick, 2015b); molecular graphics: OLEX2

(Dolomanov et al., 2009); software used to prepare material for publication: Mercury (Macrae et al., 2020).

4-(3-Carboxy-1-cyclopropyl-6-fluoro-4-oxo-1,4-dihydroquinolin-7-yl)piperazin-1-ium 2,6-dihydroxybenzoate (I)

Crystal data

$\mathrm{C}_{17} \mathrm{H}_{19} \mathrm{FN}_{3} \mathrm{O}_{3}{ }^{+} \cdot \mathrm{C}_{7} \mathrm{H}_{5} \mathrm{O}_{4}{ }^{-}$

$M_{r}=485.46$

Monoclinic, $P 2{ }_{1} / c$

$a=7.9722(5) \AA$

$b=21.2705(11) \AA$

$c=13.0860(7) \AA$

$\beta=101.805(6)^{\circ}$

$V=2172.1(2) \AA^{3}$

$Z=4$

Data collection

XtaLAB Synergy R, DW system, HyPix diffractometer

Radiation source: Rotating-anode X-ray tube, Rigaku XtaLAB Synergy-R

Mirror monochromator

Detector resolution: 10.0000 pixels $\mathrm{mm}^{-1}$

$\omega$ scans

Absorption correction: multi-scan

(CrysAlisPro; Rigaku OD, 2020)

\section{Refinement}

Refinement on $F^{2}$

Least-squares matrix: full

$R\left[F^{2}>2 \sigma\left(F^{2}\right)\right]=0.053$

$w R\left(F^{2}\right)=0.139$

$S=1.03$

4378 reflections

319 parameters

0 restraints

Primary atom site location: dual
$F(000)=1016$

$D_{\mathrm{x}}=1.485 \mathrm{Mg} \mathrm{m}^{-3}$

$\mathrm{Cu} K \alpha$ radiation, $\lambda=1.54184 \AA$

Cell parameters from 4777 reflections

$\theta=4.0-72.0^{\circ}$

$\mu=0.98 \mathrm{~mm}^{-1}$

$T=93 \mathrm{~K}$

Block, colourless

$0.23 \times 0.05 \times 0.04 \mathrm{~mm}$

$T_{\min }=0.919, T_{\max }=1.000$

15936 measured reflections

4378 independent reflections

$R_{\text {int }}=0.038$

$\theta_{\max }=76.3^{\circ}, \theta_{\min }=4.0^{\circ}$

$h=-9 \rightarrow 9$

$k=-17 \rightarrow 26$

$l=-15 \rightarrow 16$

Hydrogen site location: inferred from neighbouring sites

$\mathrm{H}$-atom parameters constrained

$w=1 /\left[\sigma^{2}\left(F_{\mathrm{o}}^{2}\right)+(0.062 P)^{2}+1.4432 P\right]$

where $P=\left(F_{\mathrm{o}}^{2}+2 F_{\mathrm{c}}^{2}\right) / 3$

$(\Delta / \sigma)_{\max }=0.001$

$\Delta \rho_{\max }=0.34 \mathrm{e} \AA^{-3}$

$\Delta \rho_{\min }=-0.41 \mathrm{e} \AA^{-3}$ 


\section{Special details}

Geometry. All esds (except the esd in the dihedral angle between two 1.s. planes) are estimated using the full covariance matrix. The cell esds are taken into account individually in the estimation of esds in distances, angles and torsion angles; correlations between esds in cell parameters are only used when they are defined by crystal symmetry. An approximate (isotropic) treatment of cell esds is used for estimating esds involving l.s. planes.

Fractional atomic coordinates and isotropic or equivalent isotropic displacement parameters $\left(\AA^{2}\right)$

\begin{tabular}{|c|c|c|c|c|}
\hline & $x$ & $y$ & $z$ & $U_{\text {iso }} * / U_{\text {eq }}$ \\
\hline F1 & $0.05295(16)$ & $0.41493(5)$ & $0.67770(9)$ & $0.0377(3)$ \\
\hline $\mathrm{O} 1$ & $0.4436(2)$ & $0.54707(8)$ & $0.16888(11)$ & $0.0432(4)$ \\
\hline $\mathrm{O} 2$ & $0.3833(2)$ & $0.44645(7)$ & $0.18852(11)$ & $0.0402(4)$ \\
\hline $\mathrm{H} 2$ & 0.347742 & 0.424196 & 0.232696 & $0.060 *$ \\
\hline $\mathrm{O} 3$ & $0.28730(19)$ & $0.41034(7)$ & $0.35014(11)$ & $0.0362(3)$ \\
\hline N1 & $0.3471(2)$ & $0.59129(8)$ & $0.46044(12)$ & $0.0314(4)$ \\
\hline N2 & $0.0934(2)$ & $0.53783(8)$ & $0.75639(13)$ & $0.0328(4)$ \\
\hline N3 & $0.2572(3)$ & $0.58568(8)$ & $0.95730(14)$ & $0.0395(4)$ \\
\hline H3A & 0.356800 & 0.595565 & 1.002153 & $0.047 *$ \\
\hline H3B & 0.169225 & 0.594851 & 0.989447 & $0.047^{*}$ \\
\hline $\mathrm{C} 1$ & $0.4001(3)$ & $0.50538(10)$ & $0.22173(15)$ & $0.0354(5)$ \\
\hline $\mathrm{C} 2$ & $0.3602(3)$ & $0.51643(10)$ & $0.32635(15)$ & $0.0327(4)$ \\
\hline $\mathrm{C} 3$ & $0.3006(2)$ & $0.46647(10)$ & $0.38290(15)$ & $0.0326(4)$ \\
\hline $\mathrm{C} 4$ & $0.2566(2)$ & $0.48427(10)$ & $0.48093(15)$ & $0.0316(4)$ \\
\hline $\mathrm{C} 5$ & $0.1856(3)$ & $0.44006(9)$ & $0.54010(15)$ & $0.0327(4)$ \\
\hline H5 & 0.172151 & 0.397574 & 0.517532 & $0.039 *$ \\
\hline C6 & $0.1363(3)$ & $0.45781(9)$ & $0.62931(15)$ & $0.0321(4)$ \\
\hline $\mathrm{C} 7$ & $0.1580(2)$ & $0.51970(9)$ & $0.67053(15)$ & $0.0313(4)$ \\
\hline $\mathrm{C} 8$ & $0.2342(2)$ & $0.56281(9)$ & $0.61296(15)$ & $0.0314(4)$ \\
\hline H8 & 0.256130 & 0.604419 & 0.638611 & $0.038 *$ \\
\hline C9 & $0.2786(2)$ & $0.54598(9)$ & $0.51851(15)$ & $0.0303(4)$ \\
\hline $\mathrm{C} 10$ & $0.3838(3)$ & $0.57567(10)$ & $0.36771(15)$ & $0.0329(4)$ \\
\hline H10 & 0.428110 & 0.607231 & 0.329187 & $0.040^{*}$ \\
\hline C11 & $0.3814(3)$ & $0.65431(9)$ & $0.50290(16)$ & $0.0338(4)$ \\
\hline H11 & 0.471754 & 0.657418 & 0.568093 & $0.041 *$ \\
\hline $\mathrm{C} 12$ & $0.2333(3)$ & $0.69888(10)$ & $0.49555(18)$ & $0.0418(5)$ \\
\hline $\mathrm{H} 12 \mathrm{~A}$ & 0.232822 & 0.727421 & 0.555245 & $0.050^{*}$ \\
\hline H12B & 0.118861 & 0.684208 & 0.459028 & $0.050^{*}$ \\
\hline C13 & $0.3671(3)$ & $0.70946(10)$ & $0.43140(17)$ & $0.0407(5)$ \\
\hline H13A & 0.334605 & 0.701423 & 0.355393 & $0.049 *$ \\
\hline H13B & 0.448510 & 0.744615 & 0.451562 & $0.049 *$ \\
\hline C14 & $0.1041(3)$ & $0.49851(10)$ & $0.84970(16)$ & $0.0357(5)$ \\
\hline H14A & 0.115360 & 0.453863 & 0.830791 & $0.043^{*}$ \\
\hline H14B & -0.002908 & 0.502959 & 0.876377 & $0.043^{*}$ \\
\hline $\mathrm{C} 15$ & $0.2556(3)$ & $0.51692(9)$ & $0.93462(16)$ & $0.0340(4)$ \\
\hline $\mathrm{H} 15 \mathrm{~A}$ & 0.250824 & 0.493237 & 0.999094 & $0.041 *$ \\
\hline H15B & 0.363113 & 0.505307 & 0.912586 & $0.041 *$ \\
\hline C16 & $0.2411(3)$ & $0.62529(10)$ & $0.86112(16)$ & $0.0364(5)$ \\
\hline H16A & 0.344474 & 0.620481 & 0.830902 & $0.044 *$ \\
\hline
\end{tabular}




$\begin{array}{lllll}\text { H16B } & 0.229875 & 0.670139 & 0.878811 & 0.044^{*} \\ \text { C17 } & 0.0831(3) & 0.60408(10) & 0.78271(15) & 0.0327(4) \\ \text { H17A } & -0.020076 & 0.611142 & 0.812303 & 0.039^{*} \\ \text { H17B } & 0.071566 & 0.629659 & 0.718442 & 0.039^{*} \\ \text { O4 } & 1.1188(2) & 0.66620(11) & 1.0984(2) & 0.0830(8) \\ \text { O5 } & 0.8981(3) & 0.62083(8) & 0.99400(18) & 0.0683(6) \\ \text { O6 } & 0.5971(2) & 0.63810(8) & 1.02051(13) & 0.0476(4) \\ \text { H6 } & 0.675991 & 0.625422 & 0.991803 & 0.071^{*} \\ \text { O7 } & 1.0721(3) & 0.74339(12) & 1.23479(19) & 0.0809(8) \\ \text { H7 } & 1.128472 & 0.712831 & 1.218955 & 0.121^{*} \\ \text { C18 } & 0.9592(3) & 0.65706(12) & 1.0688(2) & 0.0531(7) \\ \text { C19 } & 0.8411(3) & 0.68834(9) & 1.12632(16) & 0.0336(4) \\ \text { C20 } & 0.6639(3) & 0.67641(10) & 1.10169(16) & 0.0332(4) \\ \text { C21 } & 0.5540(3) & 0.70341(11) & 1.1582(2) & 0.0447(5) \\ \text { H21 } & 0.434867 & 0.694228 & 1.142088 & 0.054^{*} \\ \text { C22 } & 0.6208(4) & 0.74400(12) & 1.2386(2) & 0.0593(8) \\ \text { H22 } & 0.545515 & 0.763145 & 1.277098 & 0.071^{*} \\ \text { C23 } & 0.7929(5) & 0.75756(13) & 1.2647(2) & 0.0625(8) \\ \text { H23 } & 0.835717 & 0.785656 & 1.320376 & 0.075^{*} \\ \text { C24 } & 0.9025(3) & 0.72996(12) & 1.20927(18) & 0.0475(6)\end{array}$

Atomic displacement parameters $\left(\AA^{2}\right)$

\begin{tabular}{lllllll}
\hline & $U^{11}$ & $U^{22}$ & $U^{33}$ & $U^{12}$ & $U^{13}$ & $U^{23}$ \\
\hline $\mathrm{F} 1$ & $0.0444(7)$ & $0.0350(6)$ & $0.0334(6)$ & $-0.0065(5)$ & $0.0070(5)$ & $-0.0004(5)$ \\
O1 & $0.0555(10)$ & $0.0466(9)$ & $0.0264(7)$ & $0.0049(7)$ & $0.0058(7)$ & $0.0000(7)$ \\
O2 & $0.0470(9)$ & $0.0437(9)$ & $0.0280(7)$ & $0.0011(7)$ & $0.0030(6)$ & $-0.0068(6)$ \\
O3 & $0.0384(8)$ & $0.0360(8)$ & $0.0312(7)$ & $0.0019(6)$ & $0.0003(6)$ & $-0.0065(6)$ \\
N1 & $0.0347(9)$ & $0.0338(9)$ & $0.0239(8)$ & $0.0014(7)$ & $0.0017(7)$ & $0.0003(6)$ \\
N2 & $0.0370(9)$ & $0.0345(9)$ & $0.0254(8)$ & $-0.0019(7)$ & $0.0030(7)$ & $-0.0007(7)$ \\
N3 & $0.0477(10)$ & $0.0353(9)$ & $0.0290(9)$ & $0.0021(8)$ & $-0.0074(8)$ & $-0.0025(7)$ \\
C1 & $0.0360(11)$ & $0.0418(11)$ & $0.0255(10)$ & $0.0036(9)$ & $-0.0008(8)$ & $-0.0026(9)$ \\
C2 & $0.0300(10)$ & $0.0390(11)$ & $0.0260(9)$ & $0.0034(8)$ & $-0.0014(8)$ & $-0.0031(8)$ \\
C3 & $0.0288(9)$ & $0.0364(10)$ & $0.0288(10)$ & $0.0040(8)$ & $-0.0033(8)$ & $-0.0031(8)$ \\
C4 & $0.0290(9)$ & $0.0370(10)$ & $0.0254(9)$ & $0.0016(8)$ & $-0.0022(7)$ & $-0.0011(8)$ \\
C5 & $0.0331(10)$ & $0.0313(10)$ & $0.0298(10)$ & $0.0020(8)$ & $-0.0024(8)$ & $-0.0035(8)$ \\
C6 & $0.0304(10)$ & $0.0340(10)$ & $0.0293(10)$ & $-0.0024(8)$ & $-0.0001(8)$ & $0.0016(8)$ \\
C7 & $0.0300(9)$ & $0.0360(10)$ & $0.0253(9)$ & $0.0006(8)$ & $-0.0002(7)$ & $-0.0010(8)$ \\
C8 & $0.0308(10)$ & $0.0337(10)$ & $0.0265(9)$ & $0.0011(8)$ & $-0.0017(7)$ & $-0.0016(8)$ \\
C9 & $0.0297(10)$ & $0.0345(10)$ & $0.0242(9)$ & $0.0027(8)$ & $-0.0001(7)$ & $0.0005(8)$ \\
C10 & $0.0332(10)$ & $0.0387(11)$ & $0.0249(9)$ & $0.0031(8)$ & $0.0014(8)$ & $0.0011(8)$ \\
C11 & $0.0398(11)$ & $0.0325(10)$ & $0.0282(10)$ & $-0.0005(8)$ & $0.0053(8)$ & $-0.0009(8)$ \\
C12 & $0.0457(12)$ & $0.0372(11)$ & $0.0420(12)$ & $0.0049(9)$ & $0.0074(10)$ & $-0.0010(9)$ \\
C13 & $0.0538(13)$ & $0.0345(11)$ & $0.0328(11)$ & $0.0025(9)$ & $0.0067(9)$ & $0.0015(9)$ \\
C14 & $0.0406(11)$ & $0.0380(11)$ & $0.0275(10)$ & $-0.0058(9)$ & $0.0047(8)$ & $0.0008(8)$ \\
C15 & $0.0385(11)$ & $0.0330(10)$ & $0.0283(10)$ & $0.0013(8)$ & $0.0018(8)$ & $-0.0005(8)$ \\
C16 & $0.0410(11)$ & $0.0326(10)$ & $0.0321(10)$ & $0.0006(8)$ & $-0.0011(9)$ & $-0.0021(8)$ \\
C17 & $0.0335(10)$ & $0.0359(10)$ & $0.0269(9)$ & $0.0020(8)$ & $0.0017(8)$ & $-0.0010(8)$ \\
& & & & & &
\end{tabular}


supporting information

\begin{tabular}{lllllll} 
O4 & $0.0390(10)$ & $0.0790(14)$ & $0.140(2)$ & $0.0173(9)$ & $0.0391(12)$ & $0.0559(15)$ \\
O5 & $0.1042(17)$ & $0.0351(9)$ & $0.0862(15)$ & $0.0002(10)$ & $0.0675(13)$ & $-0.0015(10)$ \\
O6 & $0.0513(10)$ & $0.0531(10)$ & $0.0356(8)$ & $-0.0164(8)$ & $0.0027(7)$ & $-0.0101(7)$ \\
O7 & $0.0613(12)$ & $0.0922(16)$ & $0.0708(14)$ & $-0.0414(11)$ & $-0.0296(11)$ & $0.0281(13)$ \\
C18 & $0.0488(14)$ & $0.0374(13)$ & $0.082(2)$ & $0.0124(10)$ & $0.0340(14)$ & $0.0259(13)$ \\
C19 & $0.0320(10)$ & $0.0335(10)$ & $0.0344(10)$ & $-0.0010(8)$ & $0.0048(8)$ & $0.0063(8)$ \\
C20 & $0.0342(10)$ & $0.0347(10)$ & $0.0296(10)$ & $-0.0019(8)$ & $0.0036(8)$ & $0.0007(8)$ \\
C21 & $0.0394(12)$ & $0.0431(12)$ & $0.0547(14)$ & $0.0024(10)$ & $0.0172(10)$ & $0.0040(11)$ \\
C22 & $0.096(2)$ & $0.0364(13)$ & $0.0590(16)$ & $-0.0030(13)$ & $0.0477(16)$ & $-0.0036(11)$ \\
C23 & $0.108(2)$ & $0.0474(14)$ & $0.0343(12)$ & $-0.0330(15)$ & $0.0198(14)$ & $-0.0087(11)$ \\
C24 & $0.0525(14)$ & $0.0496(13)$ & $0.0341(11)$ & $-0.0194(11)$ & $-0.0056(10)$ & $0.0104(10)$ \\
\hline
\end{tabular}

Geometric parameters $\left(\AA,{ }^{\circ}\right)$

\begin{tabular}{|c|c|c|c|}
\hline $\mathrm{F} 1-\mathrm{C} 6$ & $1.359(2)$ & $\mathrm{C} 12-\mathrm{H} 12 \mathrm{~A}$ & 0.9900 \\
\hline $\mathrm{O} 1-\mathrm{C} 1$ & $1.218(3)$ & $\mathrm{C} 12-\mathrm{H} 12 \mathrm{~B}$ & 0.9900 \\
\hline $\mathrm{O} 2-\mathrm{H} 2$ & 0.8400 & $\mathrm{C} 12-\mathrm{C} 13$ & $1.503(3)$ \\
\hline $\mathrm{O} 2-\mathrm{C} 1$ & $1.325(3)$ & $\mathrm{C} 13-\mathrm{H} 13 \mathrm{~A}$ & 0.9900 \\
\hline $\mathrm{O} 3-\mathrm{C} 3$ & $1.265(2)$ & $\mathrm{C} 13-\mathrm{H} 13 \mathrm{~B}$ & 0.9900 \\
\hline $\mathrm{N} 1-\mathrm{C} 9$ & $1.405(3)$ & $\mathrm{C} 14-\mathrm{H} 14 \mathrm{~A}$ & 0.9900 \\
\hline $\mathrm{N} 1-\mathrm{C} 10$ & $1.347(3)$ & C14-H14B & 0.9900 \\
\hline $\mathrm{N} 1-\mathrm{C} 11$ & $1.455(3)$ & $\mathrm{C} 14-\mathrm{C} 15$ & $1.516(3)$ \\
\hline N2-C7 & $1.383(3)$ & $\mathrm{C} 15-\mathrm{H} 15 \mathrm{~A}$ & 0.9900 \\
\hline $\mathrm{N} 2-\mathrm{C} 14$ & $1.468(3)$ & C15-H15B & 0.9900 \\
\hline $\mathrm{N} 2-\mathrm{C} 17$ & $1.457(3)$ & C16-H16A & 0.9900 \\
\hline $\mathrm{N} 3-\mathrm{H} 3 \mathrm{~A}$ & 0.9100 & C16-H16B & 0.9900 \\
\hline $\mathrm{N} 3-\mathrm{H} 3 \mathrm{~B}$ & 0.9100 & $\mathrm{C} 16-\mathrm{C} 17$ & $1.521(3)$ \\
\hline N3-C15 & $1.492(3)$ & C17-H17A & 0.9900 \\
\hline N3-C16 & $1.498(3)$ & C17-H17B & 0.9900 \\
\hline $\mathrm{C} 1-\mathrm{C} 2$ & $1.486(3)$ & $\mathrm{O} 4-\mathrm{C} 18$ & $1.267(3)$ \\
\hline $\mathrm{C} 2-\mathrm{C} 3$ & $1.431(3)$ & $\mathrm{O} 5-\mathrm{C} 18$ & $1.263(4)$ \\
\hline $\mathrm{C} 2-\mathrm{C} 10$ & $1.369(3)$ & $\mathrm{O} 6-\mathrm{H} 6$ & 0.8400 \\
\hline $\mathrm{C} 3-\mathrm{C} 4$ & $1.448(3)$ & $\mathrm{O} 6-\mathrm{C} 20$ & $1.358(3)$ \\
\hline $\mathrm{C} 4-\mathrm{C} 5$ & $1.408(3)$ & $\mathrm{O} 7-\mathrm{H} 7$ & 0.8400 \\
\hline $\mathrm{C} 4-\mathrm{C} 9$ & $1.400(3)$ & $\mathrm{O} 7-\mathrm{C} 24$ & $1.355(3)$ \\
\hline $\mathrm{C} 5-\mathrm{H} 5$ & 0.9500 & $\mathrm{C} 18-\mathrm{C} 19$ & $1.479(3)$ \\
\hline $\mathrm{C} 5-\mathrm{C} 6$ & $1.359(3)$ & $\mathrm{C} 19-\mathrm{C} 20$ & $1.406(3)$ \\
\hline $\mathrm{C} 6-\mathrm{C} 7$ & $1.420(3)$ & $\mathrm{C} 19-\mathrm{C} 24$ & 1.409 (3) \\
\hline $\mathrm{C} 7-\mathrm{C} 8$ & $1.402(3)$ & $\mathrm{C} 20-\mathrm{C} 21$ & $1.382(3)$ \\
\hline $\mathrm{C} 8-\mathrm{H} 8$ & 0.9500 & $\mathrm{C} 21-\mathrm{H} 21$ & 0.9500 \\
\hline $\mathrm{C} 8-\mathrm{C} 9$ & $1.400(3)$ & $\mathrm{C} 21-\mathrm{C} 22$ & $1.381(4)$ \\
\hline $\mathrm{C} 10-\mathrm{H} 10$ & 0.9500 & $\mathrm{C} 22-\mathrm{H} 22$ & 0.9500 \\
\hline $\mathrm{C} 11-\mathrm{H} 11$ & 1.0000 & $\mathrm{C} 22-\mathrm{C} 23$ & $1.375(5)$ \\
\hline $\mathrm{C} 11-\mathrm{C} 12$ & $1.501(3)$ & $\mathrm{C} 23-\mathrm{H} 23$ & 0.9500 \\
\hline $\mathrm{C} 11-\mathrm{C} 13$ & $1.490(3)$ & $\mathrm{C} 23-\mathrm{C} 24$ & $1.376(4)$ \\
\hline $\mathrm{C} 1-\mathrm{O} 2-\mathrm{H} 2$ & 109.5 & $\mathrm{C} 13-\mathrm{C} 12-\mathrm{H} 12 \mathrm{~B}$ & 117.8 \\
\hline $\mathrm{C} 9-\mathrm{N} 1-\mathrm{C} 11$ & $119.24(16)$ & $\mathrm{C} 11-\mathrm{C} 13-\mathrm{C} 12$ & $60.21(15)$ \\
\hline
\end{tabular}




\begin{tabular}{|c|c|c|c|}
\hline $\mathrm{C} 10-\mathrm{N} 1-\mathrm{C} 9$ & $119.88(17)$ & $\mathrm{C} 11-\mathrm{C} 13-\mathrm{H} 13 \mathrm{~A}$ & 117.8 \\
\hline $\mathrm{C} 10-\mathrm{N} 1-\mathrm{C} 11$ & $120.86(17)$ & $\mathrm{C} 11-\mathrm{C} 13-\mathrm{H} 13 \mathrm{~B}$ & 117.8 \\
\hline $\mathrm{C} 7-\mathrm{N} 2-\mathrm{C} 14$ & $123.30(17)$ & $\mathrm{C} 12-\mathrm{C} 13-\mathrm{H} 13 \mathrm{~A}$ & 117.8 \\
\hline $\mathrm{C} 7-\mathrm{N} 2-\mathrm{C} 17$ & $120.67(17)$ & $\mathrm{C} 12-\mathrm{C} 13-\mathrm{H} 13 \mathrm{~B}$ & 117.8 \\
\hline $\mathrm{C} 17-\mathrm{N} 2-\mathrm{C} 14$ & $110.55(16)$ & $\mathrm{H} 13 \mathrm{~A}-\mathrm{C} 13-\mathrm{H} 13 \mathrm{~B}$ & 114.9 \\
\hline $\mathrm{H} 3 \mathrm{~A}-\mathrm{N} 3-\mathrm{H} 3 \mathrm{~B}$ & 107.8 & $\mathrm{~N} 2-\mathrm{C} 14-\mathrm{H} 14 \mathrm{~A}$ & 109.4 \\
\hline $\mathrm{C} 15-\mathrm{N} 3-\mathrm{H} 3 \mathrm{~A}$ & 109.0 & $\mathrm{~N} 2-\mathrm{C} 14-\mathrm{H} 14 \mathrm{~B}$ & 109.4 \\
\hline $\mathrm{C} 15-\mathrm{N} 3-\mathrm{H} 3 \mathrm{~B}$ & 109.0 & $\mathrm{~N} 2-\mathrm{C} 14-\mathrm{C} 15$ & $111.36(17)$ \\
\hline $\mathrm{C} 15-\mathrm{N} 3-\mathrm{C} 16$ & $112.86(16)$ & $\mathrm{H} 14 \mathrm{~A}-\mathrm{C} 14-\mathrm{H} 14 \mathrm{~B}$ & 108.0 \\
\hline $\mathrm{C} 16-\mathrm{N} 3-\mathrm{H} 3 \mathrm{~A}$ & 109.0 & $\mathrm{C} 15-\mathrm{C} 14-\mathrm{H} 14 \mathrm{~A}$ & 109.4 \\
\hline $\mathrm{C} 16-\mathrm{N} 3-\mathrm{H} 3 \mathrm{~B}$ & 109.0 & $\mathrm{C} 15-\mathrm{C} 14-\mathrm{H} 14 \mathrm{~B}$ & 109.4 \\
\hline $\mathrm{O} 1-\mathrm{C} 1-\mathrm{O} 2$ & $121.63(19)$ & $\mathrm{N} 3-\mathrm{C} 15-\mathrm{C} 14$ & $111.86(17)$ \\
\hline $\mathrm{O} 1-\mathrm{C} 1-\mathrm{C} 2$ & $123.19(19)$ & $\mathrm{N} 3-\mathrm{C} 15-\mathrm{H} 15 \mathrm{~A}$ & 109.2 \\
\hline $\mathrm{O} 2-\mathrm{C} 1-\mathrm{C} 2$ & $115.18(19)$ & N3-C15-H15B & 109.2 \\
\hline $\mathrm{C} 3-\mathrm{C} 2-\mathrm{C} 1$ & $121.03(18)$ & $\mathrm{C} 14-\mathrm{C} 15-\mathrm{H} 15 \mathrm{~A}$ & 109.2 \\
\hline $\mathrm{C} 10-\mathrm{C} 2-\mathrm{C} 1$ & $118.14(19)$ & $\mathrm{C} 14-\mathrm{C} 15-\mathrm{H} 15 \mathrm{~B}$ & 109.2 \\
\hline $\mathrm{C} 10-\mathrm{C} 2-\mathrm{C} 3$ & $120.83(19)$ & $\mathrm{H} 15 \mathrm{~A}-\mathrm{C} 15-\mathrm{H} 15 \mathrm{~B}$ & 107.9 \\
\hline $\mathrm{O} 3-\mathrm{C} 3-\mathrm{C} 2$ & $122.61(19)$ & $\mathrm{N} 3-\mathrm{C} 16-\mathrm{H} 16 \mathrm{~A}$ & 110.0 \\
\hline $\mathrm{O} 3-\mathrm{C} 3-\mathrm{C} 4$ & $121.90(19)$ & $\mathrm{N} 3-\mathrm{C} 16-\mathrm{H} 16 \mathrm{~B}$ & 110.0 \\
\hline $\mathrm{C} 2-\mathrm{C} 3-\mathrm{C} 4$ & $115.48(18)$ & N3-C16-C17 & $108.50(17)$ \\
\hline $\mathrm{C} 5-\mathrm{C} 4-\mathrm{C} 3$ & $120.65(18)$ & $\mathrm{H} 16 \mathrm{~A}-\mathrm{C} 16-\mathrm{H} 16 \mathrm{~B}$ & 108.4 \\
\hline $\mathrm{C} 9-\mathrm{C} 4-\mathrm{C} 3$ & $121.38(19)$ & $\mathrm{C} 17-\mathrm{C} 16-\mathrm{H} 16 \mathrm{~A}$ & 110.0 \\
\hline $\mathrm{C} 9-\mathrm{C} 4-\mathrm{C} 5$ & $117.95(18)$ & $\mathrm{C} 17-\mathrm{C} 16-\mathrm{H} 16 \mathrm{~B}$ & 110.0 \\
\hline $\mathrm{C} 4-\mathrm{C} 5-\mathrm{H} 5$ & 119.8 & $\mathrm{~N} 2-\mathrm{C} 17-\mathrm{C} 16$ & $111.57(16)$ \\
\hline $\mathrm{C} 6-\mathrm{C} 5-\mathrm{C} 4$ & $120.40(19)$ & $\mathrm{N} 2-\mathrm{C} 17-\mathrm{H} 17 \mathrm{~A}$ & 109.3 \\
\hline $\mathrm{C} 6-\mathrm{C} 5-\mathrm{H} 5$ & 119.8 & $\mathrm{~N} 2-\mathrm{C} 17-\mathrm{H} 17 \mathrm{~B}$ & 109.3 \\
\hline $\mathrm{F} 1-\mathrm{C} 6-\mathrm{C} 5$ & $117.95(18)$ & $\mathrm{C} 16-\mathrm{C} 17-\mathrm{H} 17 \mathrm{~A}$ & 109.3 \\
\hline $\mathrm{F} 1-\mathrm{C} 6-\mathrm{C} 7$ & $118.58(18)$ & $\mathrm{C} 16-\mathrm{C} 17-\mathrm{H} 17 \mathrm{~B}$ & 109.3 \\
\hline $\mathrm{C} 5-\mathrm{C} 6-\mathrm{C} 7$ & $123.34(19)$ & $\mathrm{H} 17 \mathrm{~A}-\mathrm{C} 17-\mathrm{H} 17 \mathrm{~B}$ & 108.0 \\
\hline $\mathrm{N} 2-\mathrm{C} 7-\mathrm{C} 6$ & $122.04(18)$ & $\mathrm{C} 20-\mathrm{O} 6-\mathrm{H} 6$ & 109.5 \\
\hline $\mathrm{N} 2-\mathrm{C} 7-\mathrm{C} 8$ & $121.89(18)$ & $\mathrm{C} 24-\mathrm{O} 7-\mathrm{H} 7$ & 109.5 \\
\hline $\mathrm{C} 8-\mathrm{C} 7-\mathrm{C} 6$ & $115.79(18)$ & $\mathrm{O} 4-\mathrm{C} 18-\mathrm{C} 19$ & $118.7(3)$ \\
\hline $\mathrm{C} 7-\mathrm{C} 8-\mathrm{H} 8$ & 119.2 & $\mathrm{O} 5-\mathrm{C} 18-\mathrm{O} 4$ & $122.3(3)$ \\
\hline $\mathrm{C} 9-\mathrm{C} 8-\mathrm{C} 7$ & $121.53(19)$ & $\mathrm{O} 5-\mathrm{C} 18-\mathrm{C} 19$ & $119.0(2)$ \\
\hline $\mathrm{C} 9-\mathrm{C} 8-\mathrm{H} 8$ & 119.2 & $\mathrm{C} 20-\mathrm{C} 19-\mathrm{C} 18$ & $121.1(2)$ \\
\hline $\mathrm{C} 4-\mathrm{C} 9-\mathrm{N} 1$ & $119.20(18)$ & $\mathrm{C} 20-\mathrm{C} 19-\mathrm{C} 24$ & $117.7(2)$ \\
\hline $\mathrm{C} 4-\mathrm{C} 9-\mathrm{C} 8$ & $120.89(19)$ & $\mathrm{C} 24-\mathrm{C} 19-\mathrm{C} 18$ & $121.2(2)$ \\
\hline $\mathrm{C} 8-\mathrm{C} 9-\mathrm{N} 1$ & $119.91(18)$ & $\mathrm{O} 6-\mathrm{C} 20-\mathrm{C} 19$ & $120.12(19)$ \\
\hline $\mathrm{N} 1-\mathrm{C} 10-\mathrm{C} 2$ & $123.07(19)$ & $\mathrm{O} 6-\mathrm{C} 20-\mathrm{C} 21$ & $118.6(2)$ \\
\hline $\mathrm{N} 1-\mathrm{C} 10-\mathrm{H} 10$ & 118.5 & $\mathrm{C} 21-\mathrm{C} 20-\mathrm{C} 19$ & $121.3(2)$ \\
\hline $\mathrm{C} 2-\mathrm{C} 10-\mathrm{H} 10$ & 118.5 & $\mathrm{C} 20-\mathrm{C} 21-\mathrm{H} 21$ & 120.7 \\
\hline $\mathrm{N} 1-\mathrm{C} 11-\mathrm{H} 11$ & 115.6 & $\mathrm{C} 22-\mathrm{C} 21-\mathrm{C} 20$ & $118.7(2)$ \\
\hline $\mathrm{N} 1-\mathrm{C} 11-\mathrm{C} 12$ & $118.27(18)$ & $\mathrm{C} 22-\mathrm{C} 21-\mathrm{H} 21$ & 120.7 \\
\hline $\mathrm{N} 1-\mathrm{C} 11-\mathrm{C} 13$ & $120.07(17)$ & $\mathrm{C} 21-\mathrm{C} 22-\mathrm{H} 22$ & 119.0 \\
\hline $\mathrm{C} 12-\mathrm{C} 11-\mathrm{H} 11$ & 115.6 & $\mathrm{C} 23-\mathrm{C} 22-\mathrm{C} 21$ & $122.0(2)$ \\
\hline $\mathrm{C} 13-\mathrm{C} 11-\mathrm{H} 11$ & 115.6 & $\mathrm{C} 23-\mathrm{C} 22-\mathrm{H} 22$ & 119.0 \\
\hline $\mathrm{C} 13-\mathrm{C} 11-\mathrm{C} 12$ & $60.32(15)$ & $\mathrm{C} 22-\mathrm{C} 23-\mathrm{H} 23$ & 120.4 \\
\hline $\mathrm{C} 11-\mathrm{C} 12-\mathrm{H} 12 \mathrm{~A}$ & 117.8 & $\mathrm{C} 22-\mathrm{C} 23-\mathrm{C} 24$ & $119.3(2)$ \\
\hline
\end{tabular}




\begin{tabular}{|c|c|c|c|}
\hline $\mathrm{C} 11-\mathrm{C} 12-\mathrm{H} 12 \mathrm{~B}$ & 117.8 & $\mathrm{C} 24-\mathrm{C} 23-\mathrm{H} 23$ & 120.4 \\
\hline $\mathrm{C} 11-\mathrm{C} 12-\mathrm{C} 13$ & $59.47(14)$ & $\mathrm{O} 7-\mathrm{C} 24-\mathrm{C} 19$ & $119.6(3)$ \\
\hline $\mathrm{H} 12 \mathrm{~A}-\mathrm{C} 12-\mathrm{H} 12 \mathrm{~B}$ & 115.0 & $\mathrm{O} 7-\mathrm{C} 24-\mathrm{C} 23$ & $119.3(3)$ \\
\hline $\mathrm{C} 13-\mathrm{C} 12-\mathrm{H} 12 \mathrm{~A}$ & 117.8 & $\mathrm{C} 23-\mathrm{C} 24-\mathrm{C} 19$ & $121.1(2)$ \\
\hline $\mathrm{F} 1-\mathrm{C} 6-\mathrm{C} 7-\mathrm{N} 2$ & $-1.6(3)$ & $\mathrm{C} 9-\mathrm{C} 4-\mathrm{C} 5-\mathrm{C} 6$ & $1.8(3)$ \\
\hline $\mathrm{F} 1-\mathrm{C} 6-\mathrm{C} 7-\mathrm{C} 8$ & $-175.68(16)$ & $\mathrm{C} 10-\mathrm{N} 1-\mathrm{C} 9-\mathrm{C} 4$ & $2.8(3)$ \\
\hline $\mathrm{O} 1-\mathrm{C} 1-\mathrm{C} 2-\mathrm{C} 3$ & $176.50(19)$ & $\mathrm{C} 10-\mathrm{N} 1-\mathrm{C} 9-\mathrm{C} 8$ & $-177.48(18)$ \\
\hline $\mathrm{O} 1-\mathrm{C} 1-\mathrm{C} 2-\mathrm{C} 10$ & $-4.2(3)$ & $\mathrm{C} 10-\mathrm{N} 1-\mathrm{C} 11-\mathrm{C} 12$ & $102.8(2)$ \\
\hline $\mathrm{O} 2-\mathrm{C} 1-\mathrm{C} 2-\mathrm{C} 3$ & $-2.7(3)$ & $\mathrm{C} 10-\mathrm{N} 1-\mathrm{C} 11-\mathrm{C} 13$ & $32.6(3)$ \\
\hline $\mathrm{O} 2-\mathrm{C} 1-\mathrm{C} 2-\mathrm{C} 10$ & $176.59(18)$ & $\mathrm{C} 10-\mathrm{C} 2-\mathrm{C} 3-\mathrm{O} 3$ & $-175.52(18)$ \\
\hline $\mathrm{O} 3-\mathrm{C} 3-\mathrm{C} 4-\mathrm{C} 5$ & $-4.5(3)$ & $\mathrm{C} 10-\mathrm{C} 2-\mathrm{C} 3-\mathrm{C} 4$ & $4.3(3)$ \\
\hline $\mathrm{O} 3-\mathrm{C} 3-\mathrm{C} 4-\mathrm{C} 9$ & $177.25(17)$ & $\mathrm{C} 11-\mathrm{N} 1-\mathrm{C} 9-\mathrm{C} 4$ & $-175.72(17)$ \\
\hline $\mathrm{N} 1-\mathrm{C} 11-\mathrm{C} 12-\mathrm{C} 13$ & $-110.4(2)$ & $\mathrm{C} 11-\mathrm{N} 1-\mathrm{C} 9-\mathrm{C} 8$ & $4.0(3)$ \\
\hline $\mathrm{N} 1-\mathrm{C} 11-\mathrm{C} 13-\mathrm{C} 12$ & $107.5(2)$ & $\mathrm{C} 11-\mathrm{N} 1-\mathrm{C} 10-\mathrm{C} 2$ & $177.41(18)$ \\
\hline $\mathrm{N} 2-\mathrm{C} 7-\mathrm{C} 8-\mathrm{C} 9$ & $-171.43(18)$ & $\mathrm{C} 14-\mathrm{N} 2-\mathrm{C} 7-\mathrm{C} 6$ & $42.6(3)$ \\
\hline $\mathrm{N} 2-\mathrm{C} 14-\mathrm{C} 15-\mathrm{N} 3$ & $52.0(2)$ & $\mathrm{C} 14-\mathrm{N} 2-\mathrm{C} 7-\mathrm{C} 8$ & $-143.72(19)$ \\
\hline $\mathrm{N} 3-\mathrm{C} 16-\mathrm{C} 17-\mathrm{N} 2$ & $-58.5(2)$ & $\mathrm{C} 14-\mathrm{N} 2-\mathrm{C} 17-\mathrm{C} 16$ & $61.2(2)$ \\
\hline $\mathrm{C} 1-\mathrm{C} 2-\mathrm{C} 3-\mathrm{O} 3$ & $3.8(3)$ & $\mathrm{C} 15-\mathrm{N} 3-\mathrm{C} 16-\mathrm{C} 17$ & $53.4(2)$ \\
\hline $\mathrm{C} 1-\mathrm{C} 2-\mathrm{C} 3-\mathrm{C} 4$ & $-176.44(17)$ & $\mathrm{C} 16-\mathrm{N} 3-\mathrm{C} 15-\mathrm{C} 14$ & $-51.4(2)$ \\
\hline $\mathrm{C} 1-\mathrm{C} 2-\mathrm{C} 10-\mathrm{N} 1$ & $178.08(18)$ & $\mathrm{C} 17-\mathrm{N} 2-\mathrm{C} 7-\mathrm{C} 6$ & $-166.08(18)$ \\
\hline $\mathrm{C} 2-\mathrm{C} 3-\mathrm{C} 4-\mathrm{C} 5$ & $175.74(17)$ & $\mathrm{C} 17-\mathrm{N} 2-\mathrm{C} 7-\mathrm{C} 8$ & $7.6(3)$ \\
\hline $\mathrm{C} 2-\mathrm{C} 3-\mathrm{C} 4-\mathrm{C} 9$ & $-2.5(3)$ & $\mathrm{C} 17-\mathrm{N} 2-\mathrm{C} 14-\mathrm{C} 15$ & $-56.9(2)$ \\
\hline $\mathrm{C} 3-\mathrm{C} 2-\mathrm{C} 10-\mathrm{N} 1$ & $-2.6(3)$ & $\mathrm{O} 4-\mathrm{C} 18-\mathrm{C} 19-\mathrm{C} 20$ & $-175.4(2)$ \\
\hline $\mathrm{C} 3-\mathrm{C} 4-\mathrm{C} 5-\mathrm{C} 6$ & $-176.56(18)$ & $\mathrm{O} 4-\mathrm{C} 18-\mathrm{C} 19-\mathrm{C} 24$ & $3.0(3)$ \\
\hline $\mathrm{C} 3-\mathrm{C} 4-\mathrm{C} 9-\mathrm{N} 1$ & $-0.9(3)$ & $\mathrm{O} 5-\mathrm{C} 18-\mathrm{C} 19-\mathrm{C} 20$ & $2.6(3)$ \\
\hline $\mathrm{C} 3-\mathrm{C} 4-\mathrm{C} 9-\mathrm{C} 8$ & $179.39(18)$ & $\mathrm{O} 5-\mathrm{C} 18-\mathrm{C} 19-\mathrm{C} 24$ & $-179.0(2)$ \\
\hline $\mathrm{C} 4-\mathrm{C} 5-\mathrm{C} 6-\mathrm{F} 1$ & $173.48(16)$ & $\mathrm{O} 6-\mathrm{C} 20-\mathrm{C} 21-\mathrm{C} 22$ & $-177.9(2)$ \\
\hline $\mathrm{C} 4-\mathrm{C} 5-\mathrm{C} 6-\mathrm{C} 7$ & $-2.5(3)$ & $\mathrm{C} 18-\mathrm{C} 19-\mathrm{C} 20-\mathrm{O} 6$ & $-3.4(3)$ \\
\hline $\mathrm{C} 5-\mathrm{C} 4-\mathrm{C} 9-\mathrm{N} 1$ & $-179.21(17)$ & $\mathrm{C} 18-\mathrm{C} 19-\mathrm{C} 20-\mathrm{C} 21$ & $177.1(2)$ \\
\hline $\mathrm{C} 5-\mathrm{C} 4-\mathrm{C} 9-\mathrm{C} 8$ & $1.1(3)$ & $\mathrm{C} 18-\mathrm{C} 19-\mathrm{C} 24-\mathrm{O} 7$ & $2.3(3)$ \\
\hline $\mathrm{C} 5-\mathrm{C} 6-\mathrm{C} 7-\mathrm{N} 2$ & $174.32(18)$ & $\mathrm{C} 18-\mathrm{C} 19-\mathrm{C} 24-\mathrm{C} 23$ & $-178.0(2)$ \\
\hline $\mathrm{C} 5-\mathrm{C} 6-\mathrm{C} 7-\mathrm{C} 8$ & $0.2(3)$ & $\mathrm{C} 19-\mathrm{C} 20-\mathrm{C} 21-\mathrm{C} 22$ & $1.6(3)$ \\
\hline $\mathrm{C} 6-\mathrm{C} 7-\mathrm{C} 8-\mathrm{C} 9$ & $2.7(3)$ & $\mathrm{C} 20-\mathrm{C} 19-\mathrm{C} 24-\mathrm{O} 7$ & $-179.2(2)$ \\
\hline $\mathrm{C} 7-\mathrm{N} 2-\mathrm{C} 14-\mathrm{C} 15$ & $97.0(2)$ & $\mathrm{C} 20-\mathrm{C} 19-\mathrm{C} 24-\mathrm{C} 23$ & $0.5(3)$ \\
\hline $\mathrm{C} 7-\mathrm{N} 2-\mathrm{C} 17-\mathrm{C} 16$ & $-93.5(2)$ & $\mathrm{C} 20-\mathrm{C} 21-\mathrm{C} 22-\mathrm{C} 23$ & $-0.9(4)$ \\
\hline $\mathrm{C} 7-\mathrm{C} 8-\mathrm{C} 9-\mathrm{N} 1$ & $176.92(17)$ & $\mathrm{C} 21-\mathrm{C} 22-\mathrm{C} 23-\mathrm{C} 24$ & $0.0(4)$ \\
\hline $\mathrm{C} 7-\mathrm{C} 8-\mathrm{C} 9-\mathrm{C} 4$ & $-3.4(3)$ & $\mathrm{C} 22-\mathrm{C} 23-\mathrm{C} 24-\mathrm{O} 7$ & $179.9(2)$ \\
\hline $\mathrm{C} 9-\mathrm{N} 1-\mathrm{C} 10-\mathrm{C} 2$ & $-1.1(3)$ & $\mathrm{C} 22-\mathrm{C} 23-\mathrm{C} 24-\mathrm{C} 19$ & $0.2(4)$ \\
\hline $\mathrm{C} 9-\mathrm{N} 1-\mathrm{C} 11-\mathrm{C} 12$ & $-78.7(2)$ & $\mathrm{C} 24-\mathrm{C} 19-\mathrm{C} 20-\mathrm{O} 6$ & $178.08(19)$ \\
\hline $\mathrm{C} 9-\mathrm{N} 1-\mathrm{C} 11-\mathrm{C} 13$ & $-148.89(19)$ & $\mathrm{C} 24-\mathrm{C} 19-\mathrm{C} 20-\mathrm{C} 21$ & $-1.4(3)$ \\
\hline
\end{tabular}

Hydrogen-bond geometry $\left(\AA,{ }^{\circ}\right)$

\begin{tabular}{lllll}
\hline$D-\mathrm{H}^{\prime} \cdots A$ & $D-\mathrm{H}$ & $\mathrm{H} \cdots A$ & $D \cdots A$ & $D-\mathrm{H} \cdots A$ \\
\hline $\mathrm{O} 2-\mathrm{H} 2 \cdots \mathrm{O} 3$ & 0.84 & 1.73 & $2.512(2)$ & 155 \\
$\mathrm{~N} 3-\mathrm{H} 3 A \cdots \mathrm{O} 1^{\mathrm{i}}$ & 0.91 & 2.38 & $2.977(2)$ & 123 \\
$\mathrm{~N} 3-\mathrm{H} 3 A \cdots \mathrm{O} 6$ & 0.91 & 2.09 & $2.890(2)$ & 146
\end{tabular}




$\begin{array}{lllll}\mathrm{N} 3-\mathrm{H} 3 B \cdots \mathrm{O} 4^{\mathrm{ii}} & 0.91 & 2.18 & 2.897(3) & 136 \\ \mathrm{~N} 3-\mathrm{H} 3 B \cdots \mathrm{O} 5^{\mathrm{ii}} & 0.91 & 2.24 & 3.090(3) & 155 \\ \mathrm{C} 11-\mathrm{H} 11 \cdots \mathrm{O} 3^{\mathrm{iii}} & 1.00 & 2.46 & 3.239(3) & 134 \\ \mathrm{C} 12-\mathrm{H} 12 A \cdots \mathrm{O} 4^{\mathrm{iv}} & 0.99 & 2.54 & 3.374(3) & 141 \\ \mathrm{C} 13-\mathrm{H} 13 A \cdots \mathrm{O} 7^{\mathrm{v}} & 0.99 & 2.51 & 3.193(3) & 126 \\ \mathrm{C} 14-\mathrm{H} 14 A \cdots \mathrm{F} 1 & 0.99 & 2.13 & 2.831(2) & 126 \\ \mathrm{C} 15-\mathrm{H} 15 B \cdots \mathrm{O} 1^{\mathrm{iii}} & 0.99 & 2.33 & 3.282(3) & 161 \\ \mathrm{C} 17-\mathrm{H} 17 A \cdots \mathrm{O} 5^{\mathrm{ii}} & 0.99 & 2.60 & 3.408(3) & 139 \\ \mathrm{O} 6-\mathrm{H} 6 \cdots \mathrm{O} 5 & 0.84 & 1.77 & 2.520(3) & 148 \\ \mathrm{O} 7-\mathrm{H} 7 \cdots \mathrm{O} 4 & 0.84 & 1.85 & 2.508(4) & 134 \\ \mathrm{C} 21-\mathrm{H} 21 \cdots \mathrm{O} 4^{\mathrm{ii}} & 0.95 & 2.54 & 3.488(3) & 178\end{array}$

Symmetry codes: (i) $x, y, z+1$; (ii) $x-1, y, z$; (iii) $-x+1,-y+1,-z+1$; (iv) $x-1,-y+3 / 2, z-1 / 2$; (v) $x-1, y, z-1$.

4-(3-Carboxy-1-cyclopropyl-6-fluoro-4-oxo-1,4-dihydroquinolin-7-yl) piperazin-1-ium chloride-3,5hydroxybenzoic acid-water (1/1/1) (II)

Crystal data

$\mathrm{C}_{17} \mathrm{H}_{19} \mathrm{FN}_{3} \mathrm{O}_{3}+\cdot \mathrm{C}_{7} \mathrm{H}_{6} \mathrm{O}_{4} \cdot \mathrm{Cl}^{-} \cdot \mathrm{H}_{2} \mathrm{O}$

$M_{r}=539.93$

Triclinic, $P 1$

$a=7.2165(2) \AA$

$b=8.8298(4) \AA$

$c=10.1184(3) \AA$

$\alpha=92.997(3)^{\circ}$

$\beta=95.219(2)^{\circ}$

$\gamma=111.557(4)^{\circ}$

$V=594.60(4) \AA^{3}$

Data collection

XtaLAB Synergy R, DW system, HyPix diffractometer

Radiation source: Rotating-anode X-ray tube, Rigaku XtaLAB Synergy-R

Mirror monochromator

Detector resolution: 10.0000 pixels $\mathrm{mm}^{-1}$

$\omega$ scans

Absorption correction: multi-scan

(CrysAlisPro; Rigaku OD, 2020)

\section{Refinement}

Refinement on $F^{2}$

Least-squares matrix: full

$R\left[F^{2}>2 \sigma\left(F^{2}\right)\right]=0.034$

$w R\left(F^{2}\right)=0.094$

$S=1.12$

4420 reflections

344 parameters

3 restraints

Primary atom site location: dual

Hydrogen site location: mixed
$Z=1$

$F(000)=282$

$D_{\mathrm{x}}=1.508 \mathrm{Mg} \mathrm{m}^{-3}$

$\mathrm{Cu} K \alpha$ radiation, $\lambda=1.54184 \AA$

Cell parameters from 10041 reflections

$\theta=4.4-75.8^{\circ}$

$\mu=2.00 \mathrm{~mm}^{-1}$

$T=93 \mathrm{~K}$

Block, colourless

$0.28 \times 0.2 \times 0.05 \mathrm{~mm}$

$T_{\min }=0.839, T_{\max }=1.000$

16358 measured reflections

4420 independent reflections

4323 reflections with $I>2 \sigma(I)$

$R_{\text {int }}=0.035$

$\theta_{\max }=74.5^{\circ}, \theta_{\min }=4.4^{\circ}$

$h=-9 \rightarrow 9$

$k=-10 \rightarrow 11$

$l=-12 \rightarrow 12$

$\mathrm{H}$ atoms treated by a mixture of independent and constrained refinement

$w=1 /\left[\sigma^{2}\left(F_{\mathrm{o}}^{2}\right)+(0.0589 P)^{2}+0.0709 P\right]$ where $P=\left(F_{\mathrm{o}}^{2}+2 F_{\mathrm{c}}^{2}\right) / 3$

$(\Delta / \sigma)_{\max }<0.001$

$\Delta \rho_{\max }=0.25 \mathrm{e} \AA^{-3}$

$\Delta \rho_{\min }=-0.47$ e $\AA^{-3}$

Absolute structure: Flack $x$ determined using 1889 quotients $\left[\left(I^{+}\right)-\left(I^{-}\right)\right] /\left[\left(I^{+}\right)+\left(I^{-}\right)\right]$(Parsons et al., 2013)

Absolute structure parameter: 0.011 (7) 


\section{Special details}

Geometry. All esds (except the esd in the dihedral angle between two 1.s. planes) are estimated using the full covariance matrix. The cell esds are taken into account individually in the estimation of esds in distances, angles and torsion angles; correlations between esds in cell parameters are only used when they are defined by crystal symmetry. An approximate (isotropic) treatment of cell esds is used for estimating esds involving l.s. planes.

Fractional atomic coordinates and isotropic or equivalent isotropic displacement parameters $\left(\AA^{2}\right)$

\begin{tabular}{|c|c|c|c|c|}
\hline & $x$ & $y$ & $z$ & $U_{\text {iso }} * / U_{\text {eq }}$ \\
\hline $\mathrm{Cl1}$ & $0.85144(9)$ & $0.46863(8)$ & $0.40663(7)$ & $0.02533(17)$ \\
\hline F1 & $0.4599(3)$ & $0.5185(2)$ & $0.98137(17)$ & $0.0273(4)$ \\
\hline $\mathrm{O} 1$ & $0.3224(3)$ & $-0.3703(3)$ & $1.2978(2)$ & $0.0284(5)$ \\
\hline $\mathrm{O} 2$ & $0.3642(4)$ & $-0.1499(3)$ & $1.4315(2)$ & $0.0294(5)$ \\
\hline $\mathrm{H} 2$ & 0.379572 & -0.051835 & 1.423817 & $0.044^{*}$ \\
\hline $\mathrm{O} 3$ & $0.3972(3)$ & $0.1143(3)$ & $1.3272(2)$ & $0.0280(5)$ \\
\hline N1 & $0.2840(4)$ & -0.1445 & $0.9603(2)$ & $0.0213(5)$ \\
\hline $\mathrm{N} 2$ & $0.3796(4)$ & $0.3457(3)$ & $0.7334(2)$ & $0.0210(5)$ \\
\hline N3 & 0.4407 (4) & $0.4661(3)$ & $0.4778(2)$ & $0.0233(5)$ \\
\hline $\mathrm{H} 3 \mathrm{~A}$ & 0.412202 & 0.525266 & 0.413562 & $0.028^{*}$ \\
\hline $\mathrm{H} 3 \mathrm{~B}$ & 0.528778 & 0.423848 & 0.448534 & $0.028^{*}$ \\
\hline $\mathrm{C} 1$ & $0.3370(4)$ & $-0.2274(4)$ & $1.3126(3)$ & $0.0249(6)$ \\
\hline $\mathrm{C} 2$ & $0.3276(4)$ & $-0.1342(4)$ & $1.1970(3)$ & $0.0222(6)$ \\
\hline $\mathrm{C} 3$ & $0.3655(4)$ & $0.0366(4)$ & $1.2138(3)$ & $0.0226(6)$ \\
\hline $\mathrm{C} 4$ & $0.3687(4)$ & 0.1155 (4) & 1.0907 (3) & $0.0220(6)$ \\
\hline $\mathrm{C} 5$ & $0.4158(4)$ & $0.2850(4)$ & $1.0937(3)$ & $0.0227(6)$ \\
\hline H5 & 0.444144 & 0.349621 & 1.176480 & $0.027^{*}$ \\
\hline C6 & $0.4203(4)$ & $0.3556(4)$ & $0.9773(3)$ & $0.0214(6)$ \\
\hline $\mathrm{C} 7$ & $0.3828(4)$ & $0.2667(4)$ & $0.8503(3)$ & $0.0202(6)$ \\
\hline $\mathrm{C} 8$ & 0.3389 (4) & $0.1003(4)$ & $0.8483(3)$ & $0.0212(6)$ \\
\hline H8 & 0.314618 & 0.036841 & 0.765286 & $0.025^{*}$ \\
\hline C9 & $0.3297(4)$ & $0.0241(4)$ & $0.9661(3)$ & $0.0204(6)$ \\
\hline $\mathrm{C} 10$ & $0.2882(4)$ & $-0.2168(4)$ & $1.0730(3)$ & $0.0218(6)$ \\
\hline H10 & 0.262685 & -0.330555 & 1.066643 & $0.026^{*}$ \\
\hline C11 & $0.2568(4)$ & $-0.2397(4)$ & 0.8324 (3) & $0.0219(6)$ \\
\hline H11 & 0.381927 & -0.228926 & 0.791979 & $0.026^{*}$ \\
\hline $\mathrm{C} 12$ & $0.0740(5)$ & $-0.2667(4)$ & $0.7366(3)$ & $0.0257(6)$ \\
\hline $\mathrm{H} 12 \mathrm{~A}$ & 0.087678 & -0.270948 & 0.640183 & $0.031^{*}$ \\
\hline H12B & -0.021768 & -0.217677 & 0.763308 & $0.031 *$ \\
\hline C13 & $0.0926(5)$ & $-0.4047(4)$ & 0.8124 (3) & $0.0259(6)$ \\
\hline H13A & 0.007825 & -0.440061 & 0.885206 & $0.031 *$ \\
\hline H13B & 0.117236 & -0.493315 & 0.762121 & $0.031^{*}$ \\
\hline C14 & $0.5686(4)$ & $0.4771(4)$ & 0.7139 (3) & $0.0231(6)$ \\
\hline H14A & 0.667670 & 0.429259 & 0.691921 & $0.028^{*}$ \\
\hline H14B & 0.623427 & 0.549862 & 0.797431 & $0.028^{*}$ \\
\hline C15 & $0.5351(5)$ & $0.5757(4)$ & $0.6022(3)$ & $0.0253(6)$ \\
\hline H15A & 0.447094 & 0.632955 & 0.628102 & $0.030^{*}$ \\
\hline H15B & 0.665144 & 0.659131 & 0.586453 & $0.030^{*}$ \\
\hline $\mathrm{C} 16$ & $0.2536(5)$ & $0.3306(4)$ & $0.5001(3)$ & $0.0240(6)$ \\
\hline
\end{tabular}




$\begin{array}{lllll}\text { H16A } & 0.198514 & 0.256713 & 0.417103 & 0.029^{*} \\ \text { H16B } & 0.152583 & 0.375766 & 0.523119 & 0.029^{*} \\ \text { C17 } & 0.2940(4) & 0.2351(4) & 0.6112(3) & 0.0221(6) \\ \text { H17A } & 0.167365 & 0.147006 & 0.626540 & 0.027^{*} \\ \text { H17B } & 0.388812 & 0.184215 & 0.586309 & 0.027^{*} \\ \text { O4 } & 0.8307(4) & 0.7243(3) & 0.2157(2) & 0.0292(5) \\ \text { H4 } & 0.845149 & 0.674900 & 0.282288 & 0.044^{*} \\ \text { O5 } & 0.8281(4) & 0.9108(3) & 0.3721(2) & 0.0338(5) \\ \text { O6 } & 0.9253(4) & 1.4048(3) & 0.1019(2) & 0.0282(5) \\ \text { H6 } & 0.922943 & 1.426566 & 0.183393 & 0.042^{*} \\ \text { O7 } & 0.7685(4) & 0.9471(3) & -0.2173(2) & 0.0282(5) \\ \text { H7 } & 0.769716 & 1.019031 & -0.268691 & 0.042^{*} \\ \text { C18 } & 0.8310(5) & 0.8689(4) & 0.2561(3) & 0.0245(6) \\ \text { C19 } & 0.8373(4) & 0.9759(4) & 0.1448(3) & 0.0233(6) \\ \text { C20 } & 0.8753(4) & 1.1395(4) & 0.1782(3) & 0.0230(6) \\ \text { H20 } & 0.894255 & 1.180995 & 0.269053 & 0.028^{*} \\ \text { C21 } & 0.8855(4) & 1.2429(4) & 0.0771(3) & 0.0230(6) \\ \text { C22 } & 0.8531(4) & 1.1805(4) & -0.0565(3) & 0.0229(6) \\ \text { H22 } & 0.860643 & 1.250739 & -0.125512 & 0.028^{*} \\ \text { C23 } & 0.8099(4) & 1.0150(4) & -0.0877(3) & 0.0231(6) \\ \text { C24 } & 0.8048(4) & 0.9107(4) & 0.0123(3) & 0.0243(6) \\ \text { H24 } & 0.779908 & 0.798399 & -0.009350 & 0.029^{*} \\ \text { O8 } & 0.7775(4) & 1.1327(3) & -0.4306(2) & 0.0309(5) \\ \text { H8A } & 0.799(7) & 1.230(7) & -0.457(5) & 0.046^{*} \\ \text { H8B } & 0.779(7) & 1.068(7) & -0.492(5) & 0.046^{*}\end{array}$

Atomic displacement parameters $\left(\AA^{2}\right)$

\begin{tabular}{lllllll}
\hline & $U^{11}$ & $U^{22}$ & $U^{33}$ & $U^{12}$ & $U^{13}$ & $U^{23}$ \\
\hline C11 & $0.0315(3)$ & $0.0267(3)$ & $0.0219(3)$ & $0.0151(3)$ & $0.0052(2)$ & $0.0029(2)$ \\
F1 & $0.0445(10)$ & $0.0200(9)$ & $0.0203(8)$ & $0.0152(8)$ & $0.0057(7)$ & $-0.0001(7)$ \\
O1 & $0.0376(12)$ & $0.0253(12)$ & $0.0242(11)$ & $0.0142(9)$ & $0.0011(9)$ & $0.0058(9)$ \\
O2 & $0.0419(13)$ & $0.0289(12)$ & $0.0176(10)$ & $0.0132(10)$ & $0.0032(9)$ & $0.0048(8)$ \\
O3 & $0.0426(13)$ & $0.0294(12)$ & $0.0144(9)$ & $0.0160(10)$ & $0.0048(9)$ & $0.0003(8)$ \\
N1 & $0.0270(12)$ & $0.0220(13)$ & $0.0168(12)$ & $0.0117(10)$ & $0.0024(9)$ & $-0.0002(9)$ \\
N2 & $0.0255(12)$ & $0.0232(13)$ & $0.0144(11)$ & $0.0097(10)$ & $0.0009(9)$ & $0.0008(9)$ \\
N3 & $0.0303(12)$ & $0.0286(13)$ & $0.0173(11)$ & $0.0174(10)$ & $0.0047(9)$ & $0.0046(10)$ \\
C1 & $0.0243(14)$ & $0.0325(18)$ & $0.0202(14)$ & $0.0131(12)$ & $0.0027(11)$ & $0.0050(12)$ \\
C2 & $0.0238(13)$ & $0.0266(15)$ & $0.0179(13)$ & $0.0108(11)$ & $0.0036(10)$ & $0.0041(12)$ \\
C3 & $0.0262(14)$ & $0.0272(15)$ & $0.0175(14)$ & $0.0131(12)$ & $0.0044(11)$ & $0.0038(11)$ \\
C4 & $0.0234(13)$ & $0.0268(15)$ & $0.0177(13)$ & $0.0117(11)$ & $0.0035(10)$ & $0.0007(11)$ \\
C5 & $0.0282(14)$ & $0.0232(15)$ & $0.0182(13)$ & $0.0117(12)$ & $0.0036(10)$ & $-0.0011(11)$ \\
C6 & $0.0267(14)$ & $0.0176(13)$ & $0.0206(14)$ & $0.0094(11)$ & $0.0032(11)$ & $-0.0006(11)$ \\
C7 & $0.0222(13)$ & $0.0228(15)$ & $0.0171(13)$ & $0.0105(11)$ & $0.0018(10)$ & $0.0016(11)$ \\
C8 & $0.0239(13)$ & $0.0230(15)$ & $0.0167(13)$ & $0.0095(11)$ & $0.0021(10)$ & $-0.0023(11)$ \\
C9 & $0.0210(13)$ & $0.0217(14)$ & $0.0188(14)$ & $0.0084(10)$ & $0.0030(10)$ & $0.0005(11)$ \\
C10 & $0.0242(13)$ & $0.0215(15)$ & $0.0225(14)$ & $0.0112(11)$ & $0.0033(11)$ & $0.0050(11)$ \\
C11 & $0.0291(14)$ & $0.0213(14)$ & $0.0173(13)$ & $0.0121(12)$ & $0.0024(11)$ & $-0.0009(11)$
\end{tabular}




\begin{tabular}{lllllll} 
C12 & $0.0317(15)$ & $0.0243(15)$ & $0.0213(13)$ & $0.0117(12)$ & $-0.0005(12)$ & $0.0006(11)$ \\
C13 & $0.0329(15)$ & $0.0211(15)$ & $0.0242(14)$ & $0.0110(12)$ & $0.0038(11)$ & $-0.0004(11)$ \\
C14 & $0.0281(14)$ & $0.0233(15)$ & $0.0183(13)$ & $0.0103(12)$ & $0.0021(11)$ & $0.0011(11)$ \\
C15 & $0.0335(15)$ & $0.0240(15)$ & $0.0198(14)$ & $0.0116(12)$ & $0.0050(11)$ & $0.0040(11)$ \\
C16 & $0.0291(15)$ & $0.0241(15)$ & $0.0192(13)$ & $0.0111(12)$ & $0.0006(11)$ & $0.0012(11)$ \\
C17 & $0.0264(14)$ & $0.0242(16)$ & $0.0174(13)$ & $0.0121(11)$ & $0.0006(10)$ & $0.0006(11)$ \\
O4 & $0.0432(13)$ & $0.0270(12)$ & $0.0240(11)$ & $0.0194(10)$ & $0.0077(9)$ & $0.0056(9)$ \\
O5 & $0.0540(14)$ & $0.0318(13)$ & $0.0216(11)$ & $0.0224(11)$ & $0.0074(10)$ & $0.0025(9)$ \\
O6 & $0.0423(12)$ & $0.0214(11)$ & $0.0220(10)$ & $0.0133(9)$ & $0.0046(9)$ & $-0.0007(8)$ \\
O7 & $0.0408(12)$ & $0.0264(12)$ & $0.0186(10)$ & $0.0140(10)$ & $0.0046(9)$ & $-0.0003(9)$ \\
C18 & $0.0269(14)$ & $0.0271(16)$ & $0.0230(14)$ & $0.0137(12)$ & $0.0048(11)$ & $0.0025(12)$ \\
C19 & $0.0240(13)$ & $0.0265(16)$ & $0.0216(14)$ & $0.0116(11)$ & $0.0039(11)$ & $0.0017(12)$ \\
C20 & $0.0254(13)$ & $0.0255(15)$ & $0.0196(13)$ & $0.0117(11)$ & $0.0029(11)$ & $-0.0003(11)$ \\
C21 & $0.0244(13)$ & $0.0205(14)$ & $0.0243(14)$ & $0.0092(11)$ & $0.0023(11)$ & $-0.0019(11)$ \\
C22 & $0.0249(14)$ & $0.0247(15)$ & $0.0215(14)$ & $0.0115(11)$ & $0.0045(11)$ & $0.0035(12)$ \\
C23 & $0.0240(13)$ & $0.0281(16)$ & $0.0187(14)$ & $0.0119(12)$ & $0.0023(11)$ & $-0.0010(12)$ \\
C24 & $0.0261(14)$ & $0.0259(15)$ & $0.0232(15)$ & $0.0122(12)$ & $0.0058(11)$ & $-0.0007(12)$ \\
O8 & $0.0438(13)$ & $0.0286(13)$ & $0.0212(11)$ & $0.0146(10)$ & $0.0044(9)$ & $0.0017(9)$ \\
& & & & & & \\
\hline
\end{tabular}

Geometric parameters $\left(\AA,{ }^{o}\right)$

\begin{tabular}{llll}
\hline $\mathrm{F} 1-\mathrm{C} 6$ & $1.357(4)$ & $\mathrm{C} 12-\mathrm{C} 13$ & $1.513(4)$ \\
$\mathrm{O} 1-\mathrm{C} 1$ & $1.227(4)$ & $\mathrm{C} 13-\mathrm{H} 13 \mathrm{~A}$ & 0.9900 \\
$\mathrm{O} 2-\mathrm{H} 2$ & 0.8400 & $\mathrm{C} 13-\mathrm{H} 13 \mathrm{~B}$ & 0.9900 \\
$\mathrm{O} 2-\mathrm{C} 1$ & $1.314(4)$ & $\mathrm{C} 14-\mathrm{H} 14 \mathrm{~A}$ & 0.9900 \\
$\mathrm{O} 3-\mathrm{C} 3$ & $1.263(4)$ & $\mathrm{C} 14-\mathrm{H} 14 \mathrm{~B}$ & 0.9900 \\
$\mathrm{~N} 1-\mathrm{C} 9$ & $1.397(4)$ & $\mathrm{C} 14-\mathrm{C} 15$ & $1.517(4)$ \\
$\mathrm{N} 1-\mathrm{C} 10$ & $1.339(4)$ & $\mathrm{C} 15-\mathrm{H} 15 \mathrm{~A}$ & 0.9900 \\
$\mathrm{~N} 1-\mathrm{C} 11$ & $1.463(4)$ & $\mathrm{C} 15-\mathrm{H} 15 \mathrm{~B}$ & 0.9900 \\
$\mathrm{~N} 2-\mathrm{C} 7$ & $1.407(4)$ & $\mathrm{C} 16-\mathrm{H} 16 \mathrm{~A}$ & 0.9900 \\
$\mathrm{~N} 2-\mathrm{C} 14$ & $1.468(4)$ & $\mathrm{C} 16-\mathrm{H} 16 \mathrm{~B}$ & 0.9900 \\
$\mathrm{~N} 2-\mathrm{C} 17$ & $1.467(4)$ & $\mathrm{C} 16-\mathrm{C} 17$ & $1.510(4)$ \\
$\mathrm{N} 3-\mathrm{H} 3 \mathrm{~A}$ & 0.9100 & $\mathrm{C} 17-\mathrm{H} 17 \mathrm{~A}$ & 0.9900 \\
$\mathrm{~N} 3-\mathrm{H} 3 \mathrm{~B}$ & 0.9100 & $\mathrm{C} 17-\mathrm{H} 17 \mathrm{~B}$ & 0.9900 \\
$\mathrm{~N} 3-\mathrm{C} 15$ & $1.489(4)$ & $\mathrm{O} 4-\mathrm{H} 4$ & 0.8400 \\
$\mathrm{~N} 3-\mathrm{C} 16$ & $1.484(4)$ & $\mathrm{O} 4-\mathrm{C} 18$ & $1.320(4)$ \\
$\mathrm{C} 1-\mathrm{C} 2$ & $1.475(4)$ & $\mathrm{O} 5-\mathrm{C} 18$ & $1.216(4)$ \\
$\mathrm{C} 2-\mathrm{C} 3$ & $1.428(4)$ & $\mathrm{O} 6-\mathrm{H} 6$ & 0.8400 \\
$\mathrm{C} 2-\mathrm{C} 10$ & $1.369(4)$ & $\mathrm{O} 6-\mathrm{C} 21$ & $1.356(4)$ \\
$\mathrm{C} 3-\mathrm{C} 4$ & $1.457(4)$ & $\mathrm{O} 7-\mathrm{H} 7$ & 0.8400 \\
$\mathrm{C} 4-\mathrm{C} 5$ & $1.406(4)$ & $\mathrm{O} 7-\mathrm{C} 23$ & $1.372(4)$ \\
$\mathrm{C} 4-\mathrm{C} 9$ & $1.407(4)$ & $\mathrm{C} 18-\mathrm{C} 19$ & $1.501(4)$ \\
$\mathrm{C} 5-\mathrm{H} 5$ & 0.9500 & $\mathrm{C} 19-\mathrm{C} 20$ & $1.385(4)$ \\
$\mathrm{C} 5-\mathrm{C} 6$ & $1.358(4)$ & $\mathrm{C} 19-\mathrm{C} 24$ & $1.395(4)$ \\
$\mathrm{C} 6-\mathrm{C} 7$ & $1.419(4)$ & $\mathrm{C} 20-\mathrm{H} 20$ & 0.9500 \\
$\mathrm{C} 7-\mathrm{C} 8$ & $1.384(4)$ & $\mathrm{C} 20-\mathrm{C} 21$ & $1.395(4)$ \\
$\mathrm{C} 8-\mathrm{H} 8$ & 0.9500 & $\mathrm{C} 21-\mathrm{C} 22$ & $1.399(4)$ \\
$\mathrm{C} 8-\mathrm{C} 9$ & $1.394(4)$ & $\mathrm{C} 22-\mathrm{H} 22$ & 0.9500 \\
& & &
\end{tabular}




\begin{tabular}{|c|c|c|c|}
\hline $\mathrm{C} 10-\mathrm{H} 10$ & 0.9500 & $\mathrm{C} 22-\mathrm{C} 23$ & $1.389(5)$ \\
\hline $\mathrm{C} 11-\mathrm{H} 11$ & 1.0000 & $\mathrm{C} 23-\mathrm{C} 24$ & $1.398(4)$ \\
\hline $\mathrm{C} 11-\mathrm{C} 12$ & $1.499(4)$ & $\mathrm{C} 24-\mathrm{H} 24$ & 0.9500 \\
\hline $\mathrm{C} 11-\mathrm{C} 13$ & $1.492(4)$ & $\mathrm{O} 8-\mathrm{H} 8 \mathrm{~A}$ & $0.88(6)$ \\
\hline $\mathrm{C} 12-\mathrm{H} 12 \mathrm{~A}$ & 0.9900 & $\mathrm{O} 8-\mathrm{H} 8 \mathrm{~B}$ & $0.82(6)$ \\
\hline $\mathrm{C} 12-\mathrm{H} 12 \mathrm{~B}$ & 0.9900 & & \\
\hline $\mathrm{C} 1-\mathrm{O} 2-\mathrm{H} 2$ & 109.5 & $\mathrm{C} 11-\mathrm{C} 13-\mathrm{C} 12$ & $59.9(2)$ \\
\hline $\mathrm{C} 9-\mathrm{N} 1-\mathrm{C} 11$ & $120.6(2)$ & $\mathrm{C} 11-\mathrm{C} 13-\mathrm{H} 13 \mathrm{~A}$ & 117.8 \\
\hline $\mathrm{C} 10-\mathrm{N} 1-\mathrm{C} 9$ & $119.9(3)$ & $\mathrm{C} 11-\mathrm{C} 13-\mathrm{H} 13 \mathrm{~B}$ & 117.8 \\
\hline $\mathrm{C} 10-\mathrm{N} 1-\mathrm{C} 11$ & $119.0(3)$ & $\mathrm{C} 12-\mathrm{C} 13-\mathrm{H} 13 \mathrm{~A}$ & 117.8 \\
\hline C7-N2-C14 & $115.7(2)$ & $\mathrm{C} 12-\mathrm{C} 13-\mathrm{H} 13 \mathrm{~B}$ & 117.8 \\
\hline $\mathrm{C} 7-\mathrm{N} 2-\mathrm{C} 17$ & $114.7(2)$ & $\mathrm{H} 13 \mathrm{~A}-\mathrm{C} 13-\mathrm{H} 13 \mathrm{~B}$ & 114.9 \\
\hline $\mathrm{C} 17-\mathrm{N} 2-\mathrm{C} 14$ & $111.0(2)$ & $\mathrm{N} 2-\mathrm{C} 14-\mathrm{H} 14 \mathrm{~A}$ & 109.5 \\
\hline $\mathrm{H} 3 \mathrm{~A}-\mathrm{N} 3-\mathrm{H} 3 \mathrm{~B}$ & 108.0 & $\mathrm{~N} 2-\mathrm{C} 14-\mathrm{H} 14 \mathrm{~B}$ & 109.5 \\
\hline $\mathrm{C} 15-\mathrm{N} 3-\mathrm{H} 3 \mathrm{~A}$ & 109.3 & $\mathrm{~N} 2-\mathrm{C} 14-\mathrm{C} 15$ & $110.6(2)$ \\
\hline $\mathrm{C} 15-\mathrm{N} 3-\mathrm{H} 3 \mathrm{~B}$ & 109.3 & $\mathrm{H} 14 \mathrm{~A}-\mathrm{C} 14-\mathrm{H} 14 \mathrm{~B}$ & 108.1 \\
\hline $\mathrm{C} 16-\mathrm{N} 3-\mathrm{H} 3 \mathrm{~A}$ & 109.3 & $\mathrm{C} 15-\mathrm{C} 14-\mathrm{H} 14 \mathrm{~A}$ & 109.5 \\
\hline $\mathrm{C} 16-\mathrm{N} 3-\mathrm{H} 3 \mathrm{~B}$ & 109.3 & $\mathrm{C} 15-\mathrm{C} 14-\mathrm{H} 14 \mathrm{~B}$ & 109.5 \\
\hline $\mathrm{C} 16-\mathrm{N} 3-\mathrm{C} 15$ & $111.4(2)$ & $\mathrm{N} 3-\mathrm{C} 15-\mathrm{C} 14$ & $110.2(2)$ \\
\hline $\mathrm{O} 1-\mathrm{C} 1-\mathrm{O} 2$ & $121.8(3)$ & $\mathrm{N} 3-\mathrm{C} 15-\mathrm{H} 15 \mathrm{~A}$ & 109.6 \\
\hline $\mathrm{O} 1-\mathrm{C} 1-\mathrm{C} 2$ & $121.2(3)$ & $\mathrm{N} 3-\mathrm{C} 15-\mathrm{H} 15 \mathrm{~B}$ & 109.6 \\
\hline $\mathrm{O} 2-\mathrm{C} 1-\mathrm{C} 2$ & $117.0(3)$ & $\mathrm{C} 14-\mathrm{C} 15-\mathrm{H} 15 \mathrm{~A}$ & 109.6 \\
\hline $\mathrm{C} 3-\mathrm{C} 2-\mathrm{C} 1$ & $121.3(3)$ & $\mathrm{C} 14-\mathrm{C} 15-\mathrm{H} 15 \mathrm{~B}$ & 109.6 \\
\hline $\mathrm{C} 10-\mathrm{C} 2-\mathrm{C} 1$ & $117.3(3)$ & $\mathrm{H} 15 \mathrm{~A}-\mathrm{C} 15-\mathrm{H} 15 \mathrm{~B}$ & 108.1 \\
\hline $\mathrm{C} 10-\mathrm{C} 2-\mathrm{C} 3$ & $121.4(3)$ & $\mathrm{N} 3-\mathrm{C} 16-\mathrm{H} 16 \mathrm{~A}$ & 109.5 \\
\hline $\mathrm{O} 3-\mathrm{C} 3-\mathrm{C} 2$ & $122.5(3)$ & $\mathrm{N} 3-\mathrm{C} 16-\mathrm{H} 16 \mathrm{~B}$ & 109.5 \\
\hline $\mathrm{O} 3-\mathrm{C} 3-\mathrm{C} 4$ & $122.3(3)$ & N3-C16-C17 & $110.7(2)$ \\
\hline $\mathrm{C} 2-\mathrm{C} 3-\mathrm{C} 4$ & $115.2(3)$ & $\mathrm{H} 16 \mathrm{~A}-\mathrm{C} 16-\mathrm{H} 16 \mathrm{~B}$ & 108.1 \\
\hline $\mathrm{C} 5-\mathrm{C} 4-\mathrm{C} 3$ & $120.8(3)$ & $\mathrm{C} 17-\mathrm{C} 16-\mathrm{H} 16 \mathrm{~A}$ & 109.5 \\
\hline $\mathrm{C} 5-\mathrm{C} 4-\mathrm{C} 9$ & $118.6(3)$ & $\mathrm{C} 17-\mathrm{C} 16-\mathrm{H} 16 \mathrm{~B}$ & 109.5 \\
\hline $\mathrm{C} 9-\mathrm{C} 4-\mathrm{C} 3$ & $120.6(3)$ & $\mathrm{N} 2-\mathrm{C} 17-\mathrm{C} 16$ & $109.4(2)$ \\
\hline $\mathrm{C} 4-\mathrm{C} 5-\mathrm{H} 5$ & 120.3 & $\mathrm{~N} 2-\mathrm{C} 17-\mathrm{H} 17 \mathrm{~A}$ & 109.8 \\
\hline $\mathrm{C} 6-\mathrm{C} 5-\mathrm{C} 4$ & $119.5(3)$ & $\mathrm{N} 2-\mathrm{C} 17-\mathrm{H} 17 \mathrm{~B}$ & 109.8 \\
\hline $\mathrm{C} 6-\mathrm{C} 5-\mathrm{H} 5$ & 120.3 & $\mathrm{C} 16-\mathrm{C} 17-\mathrm{H} 17 \mathrm{~A}$ & 109.8 \\
\hline $\mathrm{F} 1-\mathrm{C} 6-\mathrm{C} 5$ & $119.0(3)$ & $\mathrm{C} 16-\mathrm{C} 17-\mathrm{H} 17 \mathrm{~B}$ & 109.8 \\
\hline $\mathrm{F} 1-\mathrm{C} 6-\mathrm{C} 7$ & $117.8(2)$ & $\mathrm{H} 17 \mathrm{~A}-\mathrm{C} 17-\mathrm{H} 17 \mathrm{~B}$ & 108.2 \\
\hline $\mathrm{C} 5-\mathrm{C} 6-\mathrm{C} 7$ & $123.2(3)$ & $\mathrm{C} 18-\mathrm{O} 4-\mathrm{H} 4$ & 109.5 \\
\hline $\mathrm{N} 2-\mathrm{C} 7-\mathrm{C} 6$ & $120.4(3)$ & $\mathrm{C} 21-\mathrm{O} 6-\mathrm{H} 6$ & 109.5 \\
\hline $\mathrm{C} 8-\mathrm{C} 7-\mathrm{N} 2$ & $122.6(3)$ & $\mathrm{C} 23-\mathrm{O} 7-\mathrm{H} 7$ & 109.5 \\
\hline $\mathrm{C} 8-\mathrm{C} 7-\mathrm{C} 6$ & $116.9(3)$ & $\mathrm{O} 4-\mathrm{C} 18-\mathrm{C} 19$ & $113.1(3)$ \\
\hline $\mathrm{C} 7-\mathrm{C} 8-\mathrm{H} 8$ & 119.4 & $\mathrm{O} 5-\mathrm{C} 18-\mathrm{O} 4$ & $123.1(3)$ \\
\hline $\mathrm{C} 7-\mathrm{C} 8-\mathrm{C} 9$ & $121.1(3)$ & $\mathrm{O} 5-\mathrm{C} 18-\mathrm{C} 19$ & $123.8(3)$ \\
\hline $\mathrm{C} 9-\mathrm{C} 8-\mathrm{H} 8$ & 119.4 & $\mathrm{C} 20-\mathrm{C} 19-\mathrm{C} 18$ & $117.9(3)$ \\
\hline $\mathrm{N} 1-\mathrm{C} 9-\mathrm{C} 4$ & $119.8(2)$ & $\mathrm{C} 20-\mathrm{C} 19-\mathrm{C} 24$ & $121.7(3)$ \\
\hline $\mathrm{C} 8-\mathrm{C} 9-\mathrm{N} 1$ & $119.6(3)$ & $\mathrm{C} 24-\mathrm{C} 19-\mathrm{C} 18$ & $120.4(3)$ \\
\hline $\mathrm{C} 8-\mathrm{C} 9-\mathrm{C} 4$ & $120.7(3)$ & $\mathrm{C} 19-\mathrm{C} 20-\mathrm{H} 20$ & 120.3 \\
\hline $\mathrm{N} 1-\mathrm{C} 10-\mathrm{C} 2$ & $123.0(3)$ & $\mathrm{C} 19-\mathrm{C} 20-\mathrm{C} 21$ & $119.3(3)$ \\
\hline
\end{tabular}




\begin{tabular}{|c|c|c|c|}
\hline $\mathrm{N} 1-\mathrm{C} 10-\mathrm{H} 10$ & 118.5 & $\mathrm{C} 21-\mathrm{C} 20-\mathrm{H} 20$ & 120.3 \\
\hline $\mathrm{C} 2-\mathrm{C} 10-\mathrm{H} 10$ & 118.5 & $\mathrm{O} 6-\mathrm{C} 21-\mathrm{C} 20$ & $122.8(3)$ \\
\hline $\mathrm{N} 1-\mathrm{C} 11-\mathrm{H} 11$ & 116.2 & $\mathrm{O} 6-\mathrm{C} 21-\mathrm{C} 22$ & $117.1(3)$ \\
\hline $\mathrm{N} 1-\mathrm{C} 11-\mathrm{C} 12$ & $118.9(3)$ & $\mathrm{C} 20-\mathrm{C} 21-\mathrm{C} 22$ & $120.1(3)$ \\
\hline $\mathrm{N} 1-\mathrm{C} 11-\mathrm{C} 13$ & $117.2(2)$ & $\mathrm{C} 21-\mathrm{C} 22-\mathrm{H} 22$ & 120.2 \\
\hline $\mathrm{C} 12-\mathrm{C} 11-\mathrm{H} 11$ & 116.2 & $\mathrm{C} 23-\mathrm{C} 22-\mathrm{C} 21$ & $119.6(3)$ \\
\hline $\mathrm{C} 13-\mathrm{C} 11-\mathrm{H} 11$ & 116.2 & $\mathrm{C} 23-\mathrm{C} 22-\mathrm{H} 22$ & 120.2 \\
\hline $\mathrm{C} 13-\mathrm{C} 11-\mathrm{C} 12$ & $60.8(2)$ & $\mathrm{O} 7-\mathrm{C} 23-\mathrm{C} 22$ & $121.6(3)$ \\
\hline $\mathrm{C} 11-\mathrm{C} 12-\mathrm{H} 12 \mathrm{~A}$ & 117.8 & $\mathrm{O} 7-\mathrm{C} 23-\mathrm{C} 24$ & $117.3(3)$ \\
\hline $\mathrm{C} 11-\mathrm{C} 12-\mathrm{H} 12 \mathrm{~B}$ & 117.8 & $\mathrm{C} 22-\mathrm{C} 23-\mathrm{C} 24$ & $121.1(3)$ \\
\hline $\mathrm{C} 11-\mathrm{C} 12-\mathrm{C} 13$ & $59.4(2)$ & $\mathrm{C} 19-\mathrm{C} 24-\mathrm{C} 23$ & $118.2(3)$ \\
\hline $\mathrm{H} 12 \mathrm{~A}-\mathrm{C} 12-\mathrm{H} 12 \mathrm{~B}$ & 115.0 & $\mathrm{C} 19-\mathrm{C} 24-\mathrm{H} 24$ & 120.9 \\
\hline $\mathrm{C} 13-\mathrm{C} 12-\mathrm{H} 12 \mathrm{~A}$ & 117.8 & $\mathrm{C} 23-\mathrm{C} 24-\mathrm{H} 24$ & 120.9 \\
\hline $\mathrm{C} 13-\mathrm{C} 12-\mathrm{H} 12 \mathrm{~B}$ & 117.8 & $\mathrm{H} 8 \mathrm{~A}-\mathrm{O} 8-\mathrm{H} 8 \mathrm{~B}$ & $112(5)$ \\
\hline $\mathrm{F} 1-\mathrm{C} 6-\mathrm{C} 7-\mathrm{N} 2$ & $-2.4(4)$ & $\mathrm{C} 9-\mathrm{N} 1-\mathrm{C} 11-\mathrm{C} 13$ & $-140.3(3)$ \\
\hline $\mathrm{F} 1-\mathrm{C} 6-\mathrm{C} 7-\mathrm{C} 8$ & $-178.8(3)$ & $\mathrm{C} 9-\mathrm{C} 4-\mathrm{C} 5-\mathrm{C} 6$ & $0.7(4)$ \\
\hline $\mathrm{O} 1-\mathrm{C} 1-\mathrm{C} 2-\mathrm{C} 3$ & $-173.3(3)$ & $\mathrm{C} 10-\mathrm{N} 1-\mathrm{C} 9-\mathrm{C} 4$ & $-3.6(4)$ \\
\hline $\mathrm{O} 1-\mathrm{C} 1-\mathrm{C} 2-\mathrm{C} 10$ & $4.3(4)$ & $\mathrm{C} 10-\mathrm{N} 1-\mathrm{C} 9-\mathrm{C} 8$ & $175.5(3)$ \\
\hline $\mathrm{O} 2-\mathrm{C} 1-\mathrm{C} 2-\mathrm{C} 3$ & $5.9(4)$ & $\mathrm{C} 10-\mathrm{N} 1-\mathrm{C} 11-\mathrm{C} 12$ & $117.4(3)$ \\
\hline $\mathrm{O} 2-\mathrm{C} 1-\mathrm{C} 2-\mathrm{C} 10$ & $-176.5(3)$ & $\mathrm{C} 10-\mathrm{N} 1-\mathrm{C} 11-\mathrm{C} 13$ & $47.5(4)$ \\
\hline $\mathrm{O} 3-\mathrm{C} 3-\mathrm{C} 4-\mathrm{C} 5$ & $2.6(4)$ & $\mathrm{C} 10-\mathrm{C} 2-\mathrm{C} 3-\mathrm{O} 3$ & $178.2(3)$ \\
\hline $\mathrm{O} 3-\mathrm{C} 3-\mathrm{C} 4-\mathrm{C} 9$ & $-179.1(3)$ & $\mathrm{C} 10-\mathrm{C} 2-\mathrm{C} 3-\mathrm{C} 4$ & $-2.9(4)$ \\
\hline $\mathrm{N} 1-\mathrm{C} 11-\mathrm{C} 12-\mathrm{C} 13$ & $-106.9(3)$ & $\mathrm{C} 11-\mathrm{N} 1-\mathrm{C} 9-\mathrm{C} 4$ & $-175.7(2)$ \\
\hline $\mathrm{N} 1-\mathrm{C} 11-\mathrm{C} 13-\mathrm{C} 12$ & $109.5(3)$ & $\mathrm{C} 11-\mathrm{N} 1-\mathrm{C} 9-\mathrm{C} 8$ & $3.4(4)$ \\
\hline $\mathrm{N} 2-\mathrm{C} 7-\mathrm{C} 8-\mathrm{C} 9$ & $-175.5(3)$ & $\mathrm{C} 11-\mathrm{N} 1-\mathrm{C} 10-\mathrm{C} 2$ & $175.1(3)$ \\
\hline $\mathrm{N} 2-\mathrm{C} 14-\mathrm{C} 15-\mathrm{N} 3$ & $-55.7(3)$ & $\mathrm{C} 14-\mathrm{N} 2-\mathrm{C} 7-\mathrm{C} 6$ & $62.4(4)$ \\
\hline $\mathrm{N} 3-\mathrm{C} 16-\mathrm{C} 17-\mathrm{N} 2$ & $58.0(3)$ & $\mathrm{C} 14-\mathrm{N} 2-\mathrm{C} 7-\mathrm{C} 8$ & $-121.4(3)$ \\
\hline $\mathrm{C} 1-\mathrm{C} 2-\mathrm{C} 3-\mathrm{O} 3$ & $-4.2(4)$ & $\mathrm{C} 14-\mathrm{N} 2-\mathrm{C} 17-\mathrm{C} 16$ & $-59.9(3)$ \\
\hline $\mathrm{C} 1-\mathrm{C} 2-\mathrm{C} 3-\mathrm{C} 4$ & $174.7(3)$ & $\mathrm{C} 15-\mathrm{N} 3-\mathrm{C} 16-\mathrm{C} 17$ & $-56.1(3)$ \\
\hline $\mathrm{C} 1-\mathrm{C} 2-\mathrm{C} 10-\mathrm{N} 1$ & $-177.1(3)$ & $\mathrm{C} 16-\mathrm{N} 3-\mathrm{C} 15-\mathrm{C} 14$ & $54.5(3)$ \\
\hline $\mathrm{C} 2-\mathrm{C} 3-\mathrm{C} 4-\mathrm{C} 5$ & $-176.4(3)$ & $\mathrm{C} 17-\mathrm{N} 2-\mathrm{C} 7-\mathrm{C} 6$ & $-166.4(3)$ \\
\hline $\mathrm{C} 2-\mathrm{C} 3-\mathrm{C} 4-\mathrm{C} 9$ & $2.0(4)$ & $\mathrm{C} 17-\mathrm{N} 2-\mathrm{C} 7-\mathrm{C} 8$ & $9.8(4)$ \\
\hline $\mathrm{C} 3-\mathrm{C} 2-\mathrm{C} 10-\mathrm{N} 1$ & $0.5(4)$ & $\mathrm{C} 17-\mathrm{N} 2-\mathrm{C} 14-\mathrm{C} 15$ & $59.2(3)$ \\
\hline $\mathrm{C} 3-\mathrm{C} 4-\mathrm{C} 5-\mathrm{C} 6$ & $179.1(3)$ & $\mathrm{O} 4-\mathrm{C} 18-\mathrm{C} 19-\mathrm{C} 20$ & $-168.3(3)$ \\
\hline $\mathrm{C} 3-\mathrm{C} 4-\mathrm{C} 9-\mathrm{N} 1$ & $1.1(4)$ & $\mathrm{O} 4-\mathrm{C} 18-\mathrm{C} 19-\mathrm{C} 24$ & $11.9(4)$ \\
\hline $\mathrm{C} 3-\mathrm{C} 4-\mathrm{C} 9-\mathrm{C} 8$ & $-178.0(3)$ & $\mathrm{O} 5-\mathrm{C} 18-\mathrm{C} 19-\mathrm{C} 20$ & $11.1(5)$ \\
\hline $\mathrm{C} 4-\mathrm{C} 5-\mathrm{C} 6-\mathrm{F} 1$ & $178.0(3)$ & $\mathrm{O} 5-\mathrm{C} 18-\mathrm{C} 19-\mathrm{C} 24$ & $-168.6(3)$ \\
\hline $\mathrm{C} 4-\mathrm{C} 5-\mathrm{C} 6-\mathrm{C} 7$ & $-1.1(5)$ & $\mathrm{O} 6-\mathrm{C} 21-\mathrm{C} 22-\mathrm{C} 23$ & $-179.4(3)$ \\
\hline $\mathrm{C} 5-\mathrm{C} 4-\mathrm{C} 9-\mathrm{N} 1$ & $179.5(3)$ & $\mathrm{O} 7-\mathrm{C} 23-\mathrm{C} 24-\mathrm{C} 19$ & $-177.1(3)$ \\
\hline $\mathrm{C} 5-\mathrm{C} 4-\mathrm{C} 9-\mathrm{C} 8$ & $0.4(4)$ & $\mathrm{C} 18-\mathrm{C} 19-\mathrm{C} 20-\mathrm{C} 21$ & $178.9(3)$ \\
\hline $\mathrm{C} 5-\mathrm{C} 6-\mathrm{C} 7-\mathrm{N} 2$ & $176.7(3)$ & $\mathrm{C} 18-\mathrm{C} 19-\mathrm{C} 24-\mathrm{C} 23$ & $179.2(3)$ \\
\hline $\mathrm{C} 5-\mathrm{C} 6-\mathrm{C} 7-\mathrm{C} 8$ & $0.3(4)$ & $\mathrm{C} 19-\mathrm{C} 20-\mathrm{C} 21-\mathrm{O} 6$ & $-178.9(3)$ \\
\hline $\mathrm{C} 6-\mathrm{C} 7-\mathrm{C} 8-\mathrm{C} 9$ & $0.8(4)$ & $\mathrm{C} 19-\mathrm{C} 20-\mathrm{C} 21-\mathrm{C} 22$ & $1.4(4)$ \\
\hline $\mathrm{C} 7-\mathrm{N} 2-\mathrm{C} 14-\mathrm{C} 15$ & $-167.8(2)$ & $\mathrm{C} 20-\mathrm{C} 19-\mathrm{C} 24-\mathrm{C} 23$ & $-0.5(4)$ \\
\hline $\mathrm{C} 7-\mathrm{N} 2-\mathrm{C} 17-\mathrm{C} 16$ & $166.7(2)$ & $\mathrm{C} 20-\mathrm{C} 21-\mathrm{C} 22-\mathrm{C} 23$ & $0.3(4)$ \\
\hline $\mathrm{C} 7-\mathrm{C} 8-\mathrm{C} 9-\mathrm{N} 1$ & $179.7(3)$ & $\mathrm{C} 21-\mathrm{C} 22-\mathrm{C} 23-\mathrm{O} 7$ & $177.1(3)$ \\
\hline $\mathrm{C} 7-\mathrm{C} 8-\mathrm{C} 9-\mathrm{C} 4$ & $-1.2(4)$ & $\mathrm{C} 21-\mathrm{C} 22-\mathrm{C} 23-\mathrm{C} 24$ & $-2.2(4)$ \\
\hline
\end{tabular}


supporting information

\begin{tabular}{llll}
$\mathrm{C} 9-\mathrm{N} 1-\mathrm{C} 10-\mathrm{C} 2$ & $2.9(4)$ & $\mathrm{C} 22-\mathrm{C} 23-\mathrm{C} 24-\mathrm{C} 19$ & $2.3(4)$ \\
$\mathrm{C} 9-\mathrm{N} 1-\mathrm{C} 11-\mathrm{C} 12$ & $-70.4(4)$ & $\mathrm{C} 24-\mathrm{C} 19-\mathrm{C} 20-\mathrm{C} 21$ & $-1.3(4)$ \\
\hline
\end{tabular}

Hydrogen-bond geometry $\left(A,{ }^{\circ}\right)$

\begin{tabular}{lllll}
\hline$D-\mathrm{H} \cdots A$ & $D-\mathrm{H}$ & $\mathrm{H} \cdots A$ & $D \cdots A$ & $D-\mathrm{H} \cdots A$ \\
\hline $\mathrm{O} 2-\mathrm{H} 2 \cdots \mathrm{O} 3$ & 0.84 & 1.78 & $2.551(3)$ & 152 \\
$\mathrm{~N} 3-\mathrm{H} 3 A \cdots \mathrm{O} 1^{\mathrm{i}}$ & 0.91 & 1.75 & $2.652(3)$ & 172 \\
$\mathrm{~N} 3-\mathrm{H} 3 B \cdots \mathrm{Cl} 1$ & 0.91 & 2.30 & $3.106(3)$ & 148 \\
$\mathrm{C} 10-\mathrm{H} 10 \cdots \mathrm{F} 1^{\mathrm{ii}}$ & 0.95 & 2.46 & $3.158(4)$ & 130 \\
$\mathrm{C} 12-\mathrm{H} 12 B \cdots \mathrm{O} 7^{\mathrm{iii}}$ & 0.99 & 2.47 & $3.435(4)$ & 166 \\
$\mathrm{C} 14-\mathrm{H} 14 B \cdots \mathrm{F} 1$ & 0.99 & 2.27 & $2.927(3)$ & 123 \\
$\mathrm{C} 16-\mathrm{H} 16 B \cdots \mathrm{Cl} 1^{\mathrm{iv}}$ & 0.99 & 2.78 & $3.609(3)$ & 142 \\
$\mathrm{O} 4-\mathrm{H} 4 \cdots \mathrm{Cl1}$ & 0.84 & 2.28 & $3.082(2)$ & 160 \\
$\mathrm{O} 6-\mathrm{H} 6 \cdots \mathrm{Cl1}$ & 0.84 & 2.40 & $3.232(2)$ & 170 \\
$\mathrm{O} 7-\mathrm{H} 7 \cdots \mathrm{O} 8$ & 0.84 & 1.96 & $2.769(3)$ & 161 \\
$\mathrm{O} 8-\mathrm{H} 8 A \cdots \mathrm{C} 11^{\mathrm{i}}$ & $0.88(6)$ & $2.51(6)$ & $3.362(3)$ & $164(4)$ \\
$\mathrm{O} 8-\mathrm{H} 8 B \cdots \mathrm{O} 5^{\mathrm{vi}}$ & $0.82(6)$ & $2.05(6)$ & $2.865(4)$ & $170(5)$
\end{tabular}

Symmetry codes: (i) $x, y+1, z-1$; (ii) $x, y-1, z$; (iii) $x-1, y-1, z+1$; (iv) $x-1, y, z$; (v) $x, y+1, z$; (vi) $x, y, z-1$. 